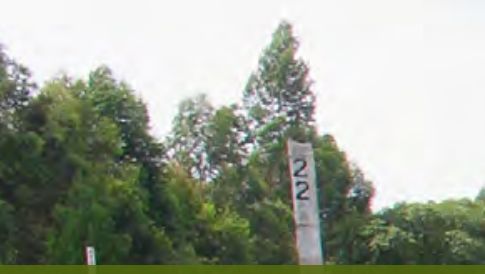

\title{
Manejo de arvenses en los sistemas
}

\section{de producción de cafe}

Luis Fernando Salazar Gutiérpez *s st

Hagar Hincapié Gómez* *

Hernán Dario Menza Franco

Fabio Alexis Yorres Angarita ****

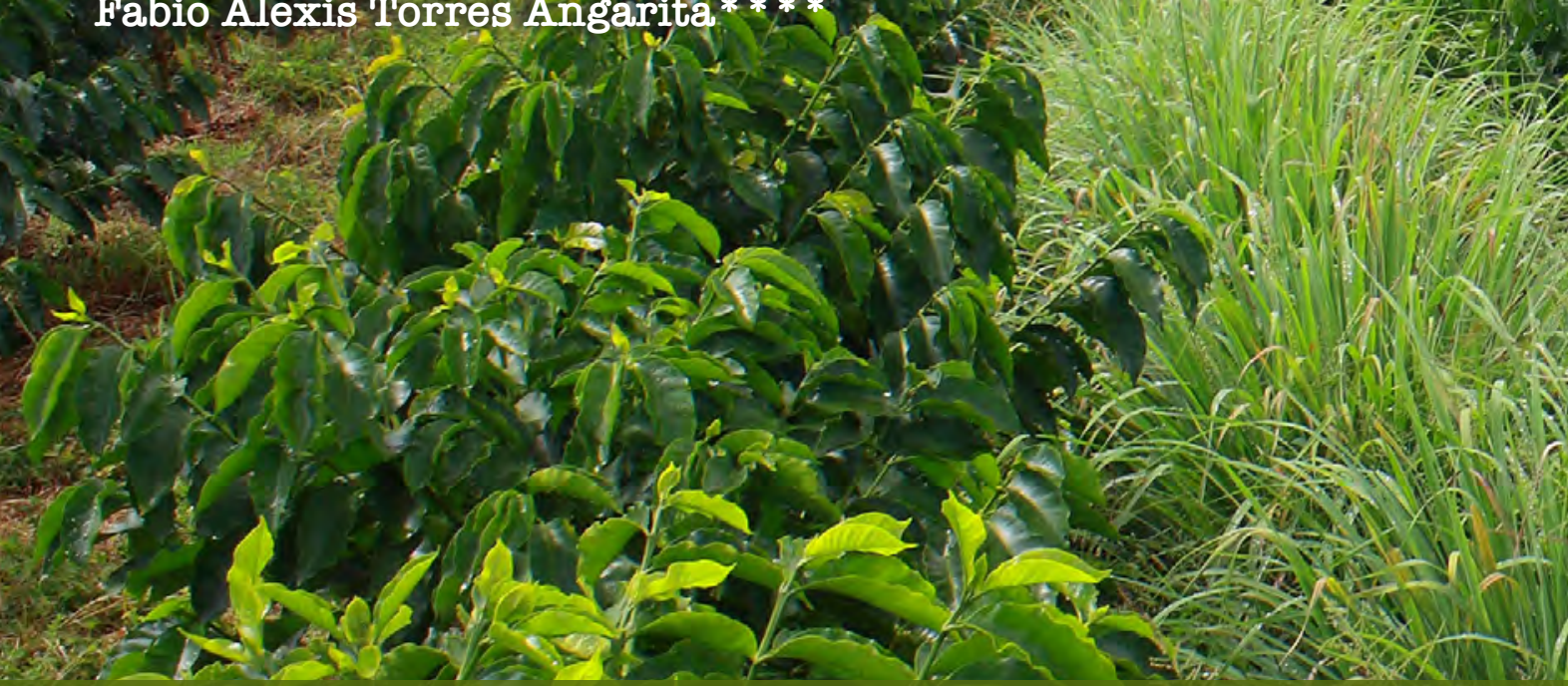

* Investigador Científico I. Disciplina de Suelos. Centro Nacional de Investigaciones de Café, Cenicafé.

ttps://orcid.org/0000-0003-2302-4825

** Ph.D. Investigador de Suelos y Aguas. Programa de Agronomía. Centro de Investigación de la Caña de Azúcar de Colombia. Cenicaña.

https://orcid.org/0000-0002-1722-791X

*** Asistente de Investigación, Disciplina de Experimentación. Centro Nacional de Investigaciones de Café, Cenicafé. https://orcid.org/0000-0003-1166-2395

**** Asistente de Investigación, Disciplina de Fitotecnia, (hasta junio de 2019). Centro Nacional de Investigaciones de Café, Cenicafé.

https://orcid.org/0000-0002-6722-7248

Salazar-Gutiérrez, L., Hincapié, F., Menza, H. D., \&e Torres, F. A. (2020). Manejo de arvenses en los sistemas de producción de café. \#n Centro Nacional de Investigaciones de Café (\$1d.), Manejo Agronómico de los Sistemas de Producción de Café (pp. 150-196). Cenicafé. https://doi.org/10.38141/10791/0002_5

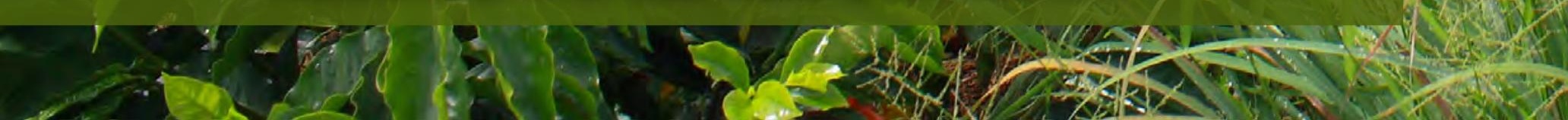


Según Gómez et al. (1985) las bases para el manejo preventivo de arvenses son:

- Uso de semilla o material vegetal certificado, libre de arvenses.

- Uso de variedades mejoradas.

- Preparación adecuada del sitio de siembra.

- Manejo de los residuos del cultivo (ramillas, hojarasca), esparciéndolos en las calles del cultivo.

- Establecimiento del cultivo en la época adecuada para asegurar disponibilidad de humedad y un crecimiento rápido y vigoroso de los cafetos.

- Manejo integrado de plagas y enfermedades.

- Fertilización según el análisis de suelo.

- Altas densidades de siembra acorde con la variedad y las condiciones agroecológicas.

- Cubrimiento de las calles del cultivo con coberturas nobles.

\section{Manejo manual de arvenses}

Es el método más recomendado en la etapa de almácigos del cultivo del café, donde deben realizarse controles muy frecuentes, para evitar la interferencia en esta etapa y así evitar el crecimiento rápido de las arvenses y, por ende, su competencia.

Este método es también recomendable para el manejo de arvenses en la zona de raíces o plato de las plantas de café, en la etapa de levante (menor a un año). En las calles del cultivo este método es viable en lotes y fincas pequeñas que dependen de mano de obra familiar. Es también recomendado en los sistemas de producción de café orgánico. Cuando existen arvenses de difícil control por otros métodos, como el caso de la especie $C$. bonariensis, el control manual es una alternativa viable, incluso para manejo en grandes extensiones. También se emplea en el control de arvenses enredaderas y parásitas.

\section{Manejo mecánico de arvenses}

Se realiza mediante herramientas de corte (manuales o motorizadas) y las más comunes en la zona cafetera son: el machete, el azadón y la guadañadora. Estas herramientas utilizadas de manera adecuada e integrada, son muy útiles para el manejo de arvenses y para evitar la erosión; este método de control debe hacerse cortando las arvenses a una altura de 3 a $5 \mathrm{~cm}$ del suelo. Se debe evitar el manejo mecánico en la zona de raíces del cultivo de café, debido al daño que se puede causar al tallo y a las raíces, lo cual favorece la infección por enfermedades al café.

\section{Manejo químico de arvenses}

Este método se basa en la utilización de herbicidas químicos para el manejo de las arvenses. Un herbicida es un producto capaz de alterar la fisiología de las plantas durante un período suficientemente largo para impedir su desarrollo normal o causar su muerte (Gómez et al., 1985). Es una herramienta utilizada para el manejo de arvenses, pero no es la única ni la más efectiva. En la actualidad el mercado mundial ofrece alrededor de 250 moléculas de herbicidas que permiten el control de la mayoría de arvenses asociadas a 
los cultivos (Valverde et al., 2000); sin embargo, desde hace más de 30 años no se producen ingredientes activos (Duke, 2012).

\section{Aspectos generales sobre herbicidas químicos}

Nombre químico. Se refiere al nombre de la molécula del ingrediente activo del herbicida.

Nombre técnico. Generalmente derivado del nombre químico, es el ingrediente activo (i.a.), puede ser una abreviatura del nombre de la molécula química o una designación arbitraria. Se usa para nombrar los herbicidas en la nomenclatura científica.

Nombre comercial. Es el nombre que le da la casa productora en el mercado y difiere según el laboratorio o casa comercial que lo produce y puede variar de un país a otro. Cuando se hace referencia a la dosis del producto comercial de un herbicida se utiliza el nombre comercial y cuando se hace referencia a la dosis del ingrediente activo debe usarse el nombre técnico.

\section{Clasificación de los herbicidas}

Según la época de aplicación los herbicidas pueden ser:

Herbicidas preemergentes. Se aplican después de la siembra del cultivo pero antes que germinen las arvenses, por ejemplo: el diuron y el oxyfluorfen son herbicidas que desnudan el suelo y tienen un alto poder residual. Estos productos forman una barrera química sobre el suelo que impide la germinación de las arvenses.

Se recomienda usar los herbicidas preemergentes como oxyfluorfen, en la etapa de almácigo y no en aplicaciones generales en el campo, ya que pueden desnudar el suelo. No obstante, pueden ser útiles para el manejo de arvenses en la zona de raíces de plantas perennes (plateo). Algunos cultivos como el café, cítricos y cacao, pueden ser susceptibles a la fitotoxicidad por la aplicación de herbicidas preemergentes como el diurón (Gómez et al., 1985).

Herbicidas postemergentes. Se aplican después de la emergencia de las arvenses. Para obtener mayor eficiencia en el control, se recomienda la aplicación antes de la etapa de floración de las arvenses. Los herbicidas postemergentes pueden ser de contacto como el paraquat y sistémicos como el glifosato o el 2,4-D amina.

Herbicidas de contacto. Son aquellos cuyo efecto sobre la arvense ocurre casi inmediatamente. El producto llega a las primeras células de las hojas o a los puntos meristemáticos, sean del tallo o de la raíz, y actúan solamente en este sitio. Un ejemplo de estos herbicidas son, el paraquat y el diquat que a la vez son desecantes de plantas, y el glufosinato de amonio que, a pesar de tener cierta acción sistémica, tiene una actividad tan rápida que actúa como un herbicida de contacto.

Herbicidas sistémicos. Son absorbidos y translocados dentro de la planta para ejercer su efecto en un lugar generalmente distinto al de penetración. Su movilidad ocurre a través del sistema vascular de la planta vía simplasto y apoplasto. Tienen la ventaja que en bajos volúmenes de aplicación y en dosis adecuadas favorecen la selectividad de arvenses, lo que permite que una población de arvenses domine sobre otras.

Según el tipo de arvenses que controlen, los herbicidas postemergentes también 
pueden clasificarse como selectivos o de amplio espectro, por ejemplo: el fluazifopbutil selectivo a arvenses de hoja angosta (gramíneas), 2,4 D amina, selectivo a arvenses de hoja ancha y el glifosato, el glufosinato de amonio y el paraquat, entre otros.

Modo de acción. Se conoce como la suma total de las respuestas anatómicas, fisiológicas y bioquímicas que constituyen la acción fitotóxica de un químico, así como la localización física y degradación molecular del herbicida en la planta (Doll, 1982).

Mecanismo de acción. Es el proceso fisiológico más específico donde actúa el herbicida para causar la muerte de la planta (Doll, 1982).

\section{Factores que afectan la aplicación de los herbicidas}

Los herbicidas son elaborados para controlar un determinado grupo de arvenses en un cultivo, durante una época específica y con una dosis que asegure la efectividad en el control, por consiguiente, la época y la dosis de aplicación dependen de varios factores relacionados con el cultivo, las especies de arvenses, el suelo y el clima, entre otros. Doll (1981) señala que el éxito del control de las arvenses mediante el uso de los herbicidas no depende únicamente del producto en sí, sino que existen otros factores de igual importancia, que en muchas ocasiones no se tienen en cuenta al momento de hacer un control químico de arvenses. Estos factores son:

- Equipos de aspersión debidamente calibrados, utilización de la boquilla y presión adecuada de acuerdo al producto que se aplique, utilizar filtros preboquillas y reguladores de presión.
- La calidad del agua usada para la preparación de la mezcla a aplicar. En general, se consideran dos aspectos: el uso de aguas calcáreas o ferruginosas (aguas duras) puede afectar la solubilidad del herbicida causando su sedimentación; esta situación se presenta principalmente con aquellos productos cuyo ingrediente activo contiene radicales ácidos y no deben utilizarse aguas que contengan sedimentos de suelo, pues la materia orgánica y las arcillas son coloides que adsorben los productos, afectando así la acción del herbicida.

- La cantidad de agua que se usa en la mezcla es un aspecto que debe tenerse en cuenta ya que, el uso de cantidades inadecuadas puede afectar la uniformidad en la aplicación o disminuir la retención de la solución por las hojas. La cantidad de agua la determina la etapa del cultivo en la cual debe hacerse la aplicación. Para aplicaciones de herbicidas preemergentes son suficientes de 150 a $250 \mathrm{~L}$ de agua por hectárea;en aplicaciones de herbicidas postemergentes se recomienda una mayor cantidad de agua, de 200 a $300 \mathrm{~L} \mathrm{ha}^{-1}$, para lograr un cubrimiento uniforme del follaje. Los herbicidas sistémicos deben aplicarse con menos cantidad de agua (200 L ha-1) y los de contacto en mayor cantidad (300 L ha-1). Para el caso del glifosato, volúmenes altos pueden reducir la efectividad del tratamiento por dilución del surfactante y retención deficiente de la solución sobre las hojas (Moreno, 1980).

- Los factores ambientales como la humedad, el viento y la temperatura afectan la eficacia de los herbicidas; por lo tanto, deben tenerse en cuenta para aplicar el producto en el momento más indicado. Debe considerarse la 
humedad del suelo, al momento de hacer la aplicación; si se aplican herbicidas pre-emergentes es necesario que el suelo esté a capacidad de campo. El rocío contribuye a la redistribución del herbicida sobre la superficie de la planta haciendo más eficiente su penetración en aplicaciones a bajo volumen; este factor influye en las aplicaciones de postemergentes de alto volumen al interferir en la retención de la mezcla del herbicida en el follaje. La lluvia puede disminuir la retención del herbicida y así disminuir su efecto. Por ejemplo, en aplicaciones de glifosato a alto volumen puede ocurrir un lavado de la mezcla, si dentro de las tres o cuatro horas siguientes a la aplicación se presentan lluvias, esto debido a la alta solubilidad del producto.

- Con referencia al viento es preferible no efectuar aplicaciones cuando la velocidad del viento sea mayor a $10 \mathrm{~km}$ $\mathrm{h}^{-1}$; también, es necesario determinar la dirección del viento para evitar que un herbicida cause toxicidad a cultivos vecinos.

- La temperatura elevada influye en las aplicaciones de herbicidas en varios aspectos:

- Aumenta la toxicidad del producto hacia el cultivo. Si se tiene un día muy caluroso y si se aplica un herbicida postemergente podría resultar más tóxico al cultivo que lo normalmente esperado, debido a la mayor evaporación del producto.

- Ocasiona marchitez de las arvenses, lo que interfiere en la translocación del herbicida (proceso de absorción foliar).
- Inactiva los herbicidas por volatilización.

- Se aumenta la actividad de algunos herbicidas postemergentes. Esto permite disminuir su dosis cuando se aplica en zonas de climas cálidos, como en el caso del 2,4-D amina. Por el contrario, las bajas temperaturas reducen la tasa de crecimiento de las arvenses, lo que hace más lenta la acción del herbicida, por lo tanto, hay que aplicar dosis mayores del producto. En general, se recomienda efectuar las aplicaciones de herbicidas cuando la temperatura está entre 15 y $32^{\circ} \mathrm{C}$.

- Para el caso del glifosato, los factores ambientales que favorecen la fotosíntesis como son la alta intensidad de la luz, la humedad adecuada en el suelo y la mayor temperatura ayudan a maximizar la translocación del herbicida, ya que el movimiento del glifosato por el floema sigue los mismos pasos y va a los mismos sitios que los azúcares producidos mediante el proceso de la fotosíntesis (Moreno, 1980).

\section{Factores que inciden en la respuesta de las arvenses a la aplicación de herbicidas}

La aplicación de un herbicida también puede fallar porque la arvense sea resistente o tolerante al herbicida, o porque se encuentre en un estado de desarrollo avanzado y el herbicida no la controle.

Concentraciones bajas de glifosato tienen el mismo efecto que las altas concentraciones, dependiendo del estado de crecimiento de la planta (Terry, 1985). 


\section{Complejo de arvenses}

Es importante tener en cuenta el complejo de arvenses existente al seleccionar el herbicida, ya que ningún herbicida selectivo controla todo tipo de arvenses.

\section{Estado de desarrollo de las arvenses}

Otro factor importante es la tolerancia de las arvenses a los herbicidas a medida que van creciendo. La época ideal para la aplicación de un postemergente es cuando las arvenses tienen de dos a tres hojas verdaderas (Hoyos, 1990) (Figura 92).

Por ejemplo, el momento más oportuno para el control de $C$. bonariensis es cuando la planta tiene una altura entre 10 a $15 \mathrm{~cm}$ (Salazar y Menza, 2018).

\section{Resistencia de las arvenses a los herbicidas}

La resistencia a herbicidas se define como la capacidad desarrollada por una población previamente susceptible para resistir la aplicación de un herbicida y completar su ciclo de vida. El desarrollo de la resistencia de una especie de arvense a un herbicida se atribuye principalmente a la presión de selección que ejerce el uso continuo del mismo sobre la población, lo que conlleva a que el control sea cada vez menos eficiente (Heap, 2005a). En la práctica, la presión de selección depende de la dosis de herbicida utilizada, su eficacia y la frecuencia de aplicación (Valverde et al., 2000).

Un obstáculo de cuidado al que se enfrenta el agricultor con el control químico de arvenses es la resistencia a los herbicidas. Valverde et al. (2000) afirman que, si no se establecen estrategias sostenibles de manejo integrado de arvenses, la utilidad futura de los herbicidas está seriamente amenazada, debido a que la adopción del manejo integrado de arvenses ha sido limitada a nivel mundial. En algunos predios productivos de la zona cafetera colombiana se evidencian factores del manejo de las arvenses que pueden generar casos potenciales de resistencia a los herbicidas, como son: el uso de un herbicida con un solo mecanismo de acción, la alta frecuencia en la aplicación del mismo herbicida por más de 30 años, las aplicaciones en forma generalizada, la calibración poco técnica de equipos y la utilización del método químico como única alternativa de control.
Figura 92.

Estado biológico para el control eficaz de arvenses (Hoyos, 1990).

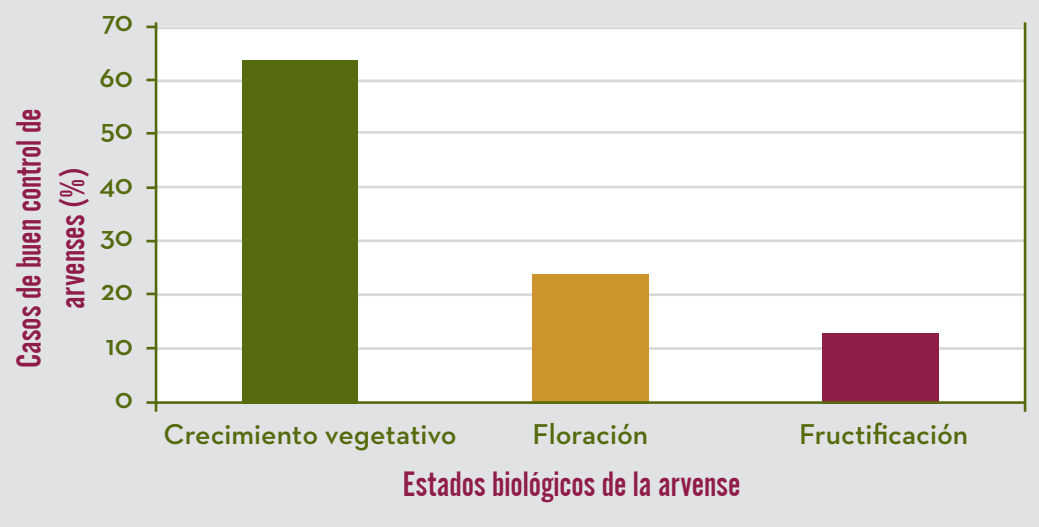




\section{¿Sabia que?}

En el año 2005, la International

Survey of Herbicide Resistant

Weeds registró 292 biotipos de arvenses resistentes a herbicidas, correspondientes

a 174 especies diferentes (104 dicotiledóneas y 70 monocotiledóneas), en 59 países (Heap, 2005b). En el año 2018, registró 490 biotipos de arvenses resistentes, 254 especies diferentes, en 70 países (Heap, 2018). Desde 1996 hasta el 2018 , se han reportado en el mundo 41 especies de arvenses y entre ellas están Conyza bonariensis y Eleusine indica, presentes en cafetales en Colombia, las cuales son resistentes al glifosato.
Prevención y manejo de la resistencia. Dentro de las recomendaciones para prevenir la resistencia pueden citarse:

- El control de arvenses mediante la integración de métodos manuales, mecánicos y químicos de forma conjunta, sin dependencia excesiva en cualquiera de ellos (Njoroge, 1994b).

- Mezcla y rotación de herbicidas con diferentes mecanismos de acción (Wrubel y Gressel, 1994), es decir, cambiar la molécula del herbicida y no solamente el nombre comercial.

Cuando ya se ha comprobado la resistencia de una arvense a un determinado herbicida, es necesario (Njoroge, 1994b, Menza y Salazar, 2007):

- Evitar el uso del herbicida al que se ha confirmado la resistencia, salvo que se utilice en mezcla con otros herbicidas de diferente mecanismo de acción.

- No incrementar la dosis del herbicida al que se ha confirmado la resistencia, ya que se acelera aún más el desarrollo de la misma y cada vez se necesitará de una dosis mayor.

- Limitar el movimiento de las poblaciones resistentes entre los campos, limpiando la maquinaria o herramientas para evitar la transferencia de semillas.

- Emplear otros herbicidas con mecanismo de acción diferente al herbicida que se le confirmó la resistencia.

- Implementar un programa de manejo integrado de arvenses para evitar 
Figura 93.

Control de biotipos de $E$. indica con dosis crecientes de glifosato, 21 días después de realizada la aplicación. Plantas bajo condiciones controladas en casa de mallas.

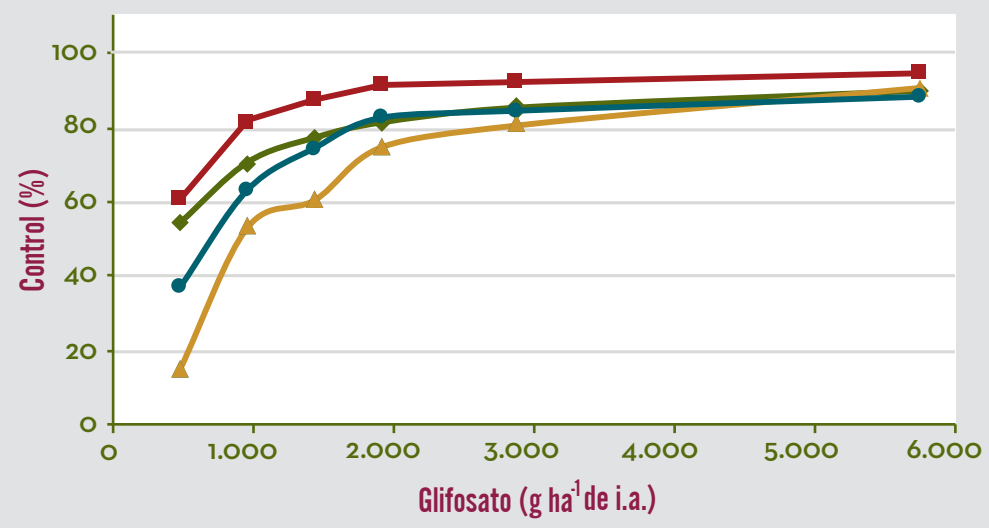

- Biotipo finca $D \prec$ Biotipo finca $A \multimap$ Biotipo finca $B \multimap$ Biotipo finca $C$

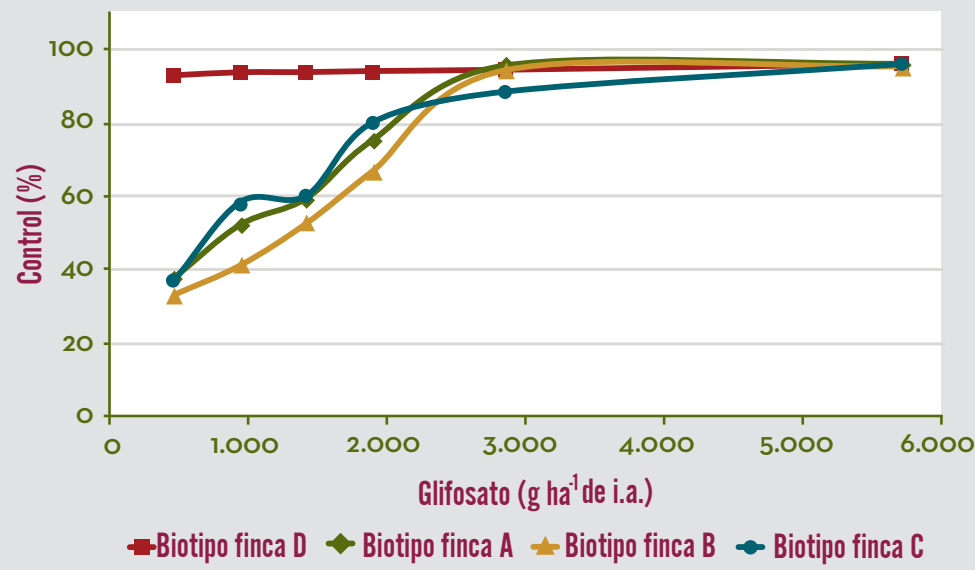

Tigura 94.

Control en biotipos de C. bonariensis con dosis crecientes de glifosato, 21 días después de realizada la aplicación. Plantas bajo condiciones controladas en casa de mallas. que otras especies sigan adquiriendo resistencia a los herbicidas.

\section{Daños a cultivos por fitotoxicidad}

Cuando el manejo químico de las arvenses no se hace en forma técnica con las debidas precauciones, pueden ocasionarse serios problemas a cultivos, lo que se ve reflejado en la disminución de la producción. En maíz, por ejemplo, la fitotoxicidad por deriva de glifosato puede causar una disminución del rendimiento mayor al 60\%, en algodón hasta del $86 \%$ y en arroz con herbicidas distintos a glifosato, la disminución puede llegar hasta el 40\% (Braverman, 1998; Rowland et al., 1999; Matthews et al., 1998).

En cultivos de café cuando se presenta fitotoxicidad por herbicidas (Figura 95) es muy poco o casi nada lo que puede hacerse para corregirlo, por lo tanto, es importante tomar las precauciones necesarias para reducir los riesgos de daño. 
Los daños al cultivo de café debido a los herbicidas generalmente se deben a varios factores, los cuales deben ser tenidos en cuenta antes de la aplicación (Galvis y Salazar, 2009). Como por ejemplo:

- Hacer aplicación de herbicidas sobre arvenses en estado avanzado de desarrollo, las cuales sobrepasen la altura del cultivo.

- Aplicación de herbicidas bajo condiciones adversas de clima (vientos).

- Aplicaciones de herbicida en forma generalizada y reiterada.

- Equipos de aplicación mal calibrados y en mal estado.

- Sobredosificación del producto.

- Mezcla inadecuada de herbicidas y coadyuvantes.

- Deficiencia o ausencia de mantenimiento de los equipos de aplicación.

- Operarios mal capacitados o sin conocimiento de la labor.

La fitotoxicidad en café por derivas de glifosato, causa defoliación, retraso del crecimiento y síntomas de deficiencia nutricional (Galvis y Salazar, 2009).

\section{Eficacia y persistencia del manejo de arvenses en cafetales con diferentes herbicidas}

Entre los años 1960 y 1980 la exploración de nuevas moléculas para el control de arvenses en café era frecuente (Gómez et al. 1987). Desde la llegada de glifosato en los años 1970 a 1980 disminuyó la búsqueda de otras moléculas para el control de arvenses en el cultivo del café. Actualmente, con la evolución de la resistencia de las arvenses a los herbicidas (Heap, 2018) se observa que las herramientas son escasas. Desde la década de 1980 los herbicidas más empleados en la zona cafetera han sido: glifosato $(84,7 \%)$, paraquat $(13,1 \%)$ y oxyfluorfen (10,9\%) (Herrera, 1983). Entre tanto, Tabares (1989) encontró que en el $74 \%$ del área con cafetales tecnificados ya aplicaban herbicidas al finalizar la década de 1980, lo que plantea una adopción generalizada de estos por los caficultores de áreas medianas y grandes en la región cafetera central de Colombia.

Las investigaciones de Cenicafé han demostrado que el glifosato es el herbicida más eficiente para el manejo de arvenses en cultivos de café debido a su alta persistencia y su eficacia hasta del 90\% (Tabla 30); sin embargo, su uso generalizado e irracional puede ocasionar erosión, contaminación del ambiente, fitotoxicidad a los cultivos, toxicidad al hombre y la resistencia de arvenses al mismo.

\section{Herbicidas actualmente recomendados en el cultivo del café en Colombia}

\section{Postemergentes de amplio espectro}

Acción sistémica: Glifosato: Sal isopropilamina $480 \mathrm{~g} \mathrm{~L}^{-1}$ en dosis de 2,0 a 3,0 L ha-1.

Acción de contacto: Glufosinato de amonio $150 \mathrm{~g} \mathrm{~L}^{-1}$, en dosis 1,5 $\mathrm{L} \mathrm{ha}^{-1}$. Paraquat: Dosis de 1,0 a 1,5 L ha-1. 
Figura 95.

Síntomas de

fitotoxicidad por

glifosato en café. a)

Después de 15 días

de la aplicación;

b) Después de

90 días de la

aplicación (Galvis

y Salazar, 2009).

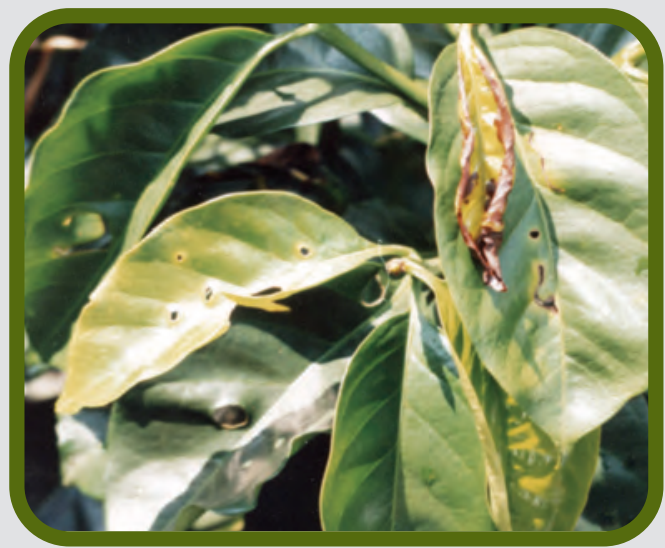

a.

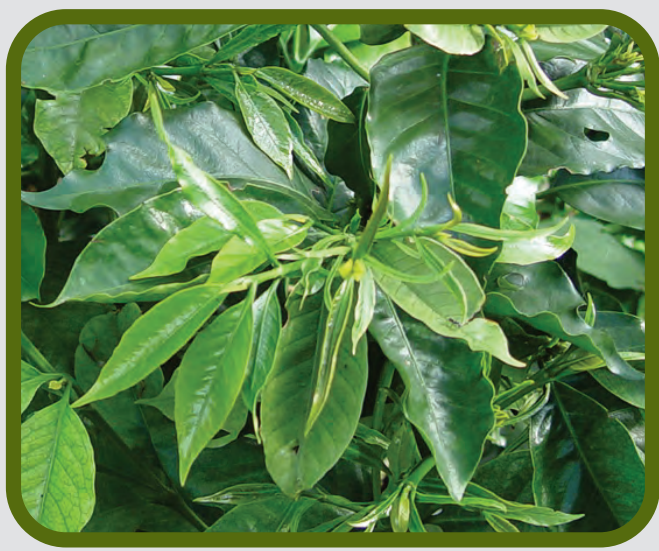

b.

Tabla 30.

Eficacia y persistencia del control de arvenses en el cultivo de café con diferentes herbicidas químicos (Hoyos, 1990).

\begin{tabular}{|c|c|c|c|c|}
\hline \multirow{3}{*}{ Tratamiento } & \multicolumn{3}{|c|}{ Dosis de producto comercial } & \multirow{3}{*}{$\begin{array}{c}\text { Persistencia } \\
\text { (promedio días) }\end{array}$} \\
\hline & 0,75 & 1,00 & 1,25 & \\
\hline & \multicolumn{3}{|c|}{ Eficiencia de control (\%) } & \\
\hline glifosato & 77 & 89 & 91 & 81 \\
\hline glifosato $+1 \%$ de urea & 80 & 82 & 89 & 76 \\
\hline paraquat 200 + diurón 800 & 76 & 81 & 77 & 53 \\
\hline glifosato + 2,4-D amina & 58 & 69 & 73 & 53 \\
\hline paraquat & 68 & 70 & 77 & 46 \\
\hline paraquat 200 + diurón 100 & 67 & 69 & 68 & 45 \\
\hline
\end{tabular}

\section{Postemergentes selectivos}

Hoja ancha: 2.4-D Amina $480 \mathrm{~g} \mathrm{~L}^{-1}$ en dosis de 2,5 L ha-1. Casos puntuales en café para arvenses resistentes o tolerantes a glifosato como $C$. bonariensis y arvenses enredaderas.

Gramicidas: Fluazifop - p - butil: 1,0 a 1,5 L ha-1.

\section{Preemergentes}

Para uso únicamente en la zona de raíces o platos del cultivo del café
Oxyfluorfen: $240 \mathrm{~g} \mathrm{~L}^{-1}$, en dosis de 3,0 a 4,0 L ha ${ }^{-1}$.

Diurón: $800 \mathrm{~g} \mathrm{~kg}^{-1}$ de i.a, en dosis de 2,0 $\mathrm{kg} \mathrm{ha}^{-1}$.

Pendimetalina: $400 \mathrm{~g} \mathrm{~L}^{-1}$, en dosis de 2,0 L ha ${ }^{-1}$.

\section{Control químico de arvenses resistentes a glifosato o de dificil manejo en cafetales}

Las arvenses E. indica (pategallina) y $C$. bonariensis (venadillo) se han encontrado resistentes a glifosato en la zona cafetera 
y los caficultores han manifestado la dificultad para su control químico. También se ha observado una frecuencia mayor de Emilia sonchifolia (emilia) en las fincas donde realizan aplicaciones de este herbicida en forma continua y generalizada (Menza, 2006; Menza y Salazar, 2006).

En una investigación realizada en Cenicafé, se evaluaron tres herbicidas con mecanismos de acción diferentes al del glifosato, así como un coadyuvante para mejorar la eficiencia de los herbicidas; el glifosato se evaluó como tratamiento testigo o punto de referencia. Los herbicidas fluazifop - $p$ - butil y glufosinato de amonio fueron las alternativas químicas (diferentes al glifosato) más eficientes para el control de E. indica (Tabla 31). El herbicida 2, 4-D amina, el herbicida glufosinato de amonio y la mezcla glifosato + 2, 4-D amina, fueron las alternativas químicas más eficientes para el control de C. bonariensis (Tabla 32). El glifosato es el herbicida con el cual se obtuvo un eficiente control (> 90\%) de E. sonchifolia. La mezcla de glifosato con el 2, 4-D amina, puede ser otra alternativa para el control de $E$. sonchifolia en condiciones de la Región Cafetera Central (Tabla 32).

Existen otras alternativas con mecanismos de acción diferentes al glifosato para el control eficiente de las arvenses estudiadas, que permiten la prevención y el manejo de posibles casos de resistencia. Su utilización puede incluirse preferiblemente dentro de un programa de manejo integrado de arvenses.

\section{Panicum laxum conocido como paja} morada por los caficultores de la zona cafetera Central, es una arvense considerada de interferencia alta en el cultivo del café en Colombia. Los mismos agricultores han reportado la dificultad para su manejo con glifosato. Con fluazifop -p- butil a los 15 días después de la aplicación se alcanzó el valor promedio más alto de control (89\%), lo cual coincidió con la mejor calificación por parte del agricultor como buen control (B) (Tabla 33). El paraquat fue el herbicida de contacto más eficaz a través del tiempo, al lograr un control de la arvense hasta del $74 \%$, recibió una calificación buena (B) por parte del agricultor ocho días después de la aplicación (Salazar e Hincapié, 2013).

En el cultivo del café en condiciones de la zona cafetera central, en la Estación Experimental La Catalina, el control de arvenses con glufosinato de amonio 150 $\mathrm{g} \mathrm{L}^{-1}$ no presentó diferencias estadísticas respecto al control alcanzado con glifosato hasta los 28 días después de su aplicación. El tratamiento más persistente en el tiempo fue el glifosato, debido a su acción sistémica y de amplio espectro.

\section{Tabla 31.}

Control de E. indica con los diferentes tratamientos de herbicidas (Menza, 2006; Menza y Salazar, 2006).

\begin{tabular}{|c|c|c|}
\hline Tratamientos herbicidas & Control (\%) & ( \\
\hline glifosato & $54,8 \mathrm{c}$ & 38,2 \\
\hline fluazifop - - butil & $75,2 \mathrm{ab}$ & 13,9 \\
\hline glufosinato de amonio & $62,5 \mathrm{bc}$ & 9,7 \\
\hline glifosato + coadyuvante & $61,0 \mathrm{bc}$ & 51,8 \\
\hline fluazifop -p- butil + coadyuvante & $88,0 \mathrm{a}$ & 2,8 \\
\hline glufosinato de amonio + coadyuvante & $77,8 \mathrm{ab}$ & 12,8 \\
\hline
\end{tabular}

*Letras distintas indican diferencias entre promedios según la prueba Duncan al $5 \%$. 
Tabla 32.

Control de Conyza bonariensis y Emilia sonchifolia con los diferentes tratamientos de herbicidas (Menza, 2006; Menza y Salazar, 2006).

\begin{tabular}{|c|c|c|c|c|}
\hline \multirow{2}{*}{ Tratamientos herbicidas } & \multicolumn{2}{|c|}{ Conyza bonariensis } & \multicolumn{2}{|c|}{ Emilia sonchifolia } \\
\cline { 2 - 5 } & Control (\%)* & $\mathbf{C . V}$. & Control (\%) & C.V. \\
\hline glifosato & $6,8 \mathrm{~b}$ & 65,6 & $91,5 \mathrm{a}$ & 2,7 \\
\hline 2, 4-D amina & $96,7 \mathrm{a}$ & 4,8 & $30,2 \mathrm{c}$ & 80,3 \\
\hline glufosinato de amonio & $79,6 \mathrm{a}$ & 38,0 & $40,1 \mathrm{c}$ & 87,1 \\
\hline glifosato + 2, 4-D amina & $96,8 \mathrm{a}$ & 2,1 & $70,9 \mathrm{ab}$ & 5,8 \\
\hline glifosato + coadyuvante & $11,5 \mathrm{~b}$ & 60,5 & $84,2 \mathrm{ab}$ & 13,3 \\
\hline 2, 4-D amina + coadyuvante & $94,3 \mathrm{a}$ & 3,7 & $56,3 \mathrm{bc}$ & 36,9 \\
\hline glufosinato de amonio + coadyuvante & $87,2 \mathrm{a}$ & 22,5 & $31,8 \mathrm{c}$ & 54,9 \\
\hline glifosato + 2, 4-D amina + coadyuvante & $95,1 \mathrm{a}$ & 2,2 & $66,8 \mathrm{ab}$ & 35,5 \\
\hline
\end{tabular}

*Letras distintas indican diferencias entre promedios según la prueba Duncan al 5\%. C.V.:coeficiente de variación

Tabla 33.

Calificación del control de paja morada Panicum laxum por el agricultor.

\begin{tabular}{|c|c|c|c|}
\hline & $\mathbf{8} \mathbf{~ d d a}$ & $\mathbf{1 5} \mathbf{~ d d a}$ & $\mathbf{2 1} \mathbf{~ d d a}$ \\
\hline glifosato (selector) & $\mathrm{M}$ & $\mathrm{M}$ & $\mathrm{M}$ \\
\hline fluazifop -p- butil & $\mathrm{R}$ & $\mathrm{B}$ & $\mathrm{B}$ \\
\hline diquat & $\mathrm{R}$ & $\mathrm{M}$ & $\mathrm{M}$ \\
\hline glufosinato de amonio & $\mathrm{B}$ & $\mathrm{M}$ & $\mathrm{R}$ \\
\hline paraquat & $\mathrm{B}$ & $\mathrm{R}$ & $\mathrm{M}$ \\
\hline glifosato (aspersión) & $\mathrm{M}$ & $\mathrm{M}$ & \\
\hline E: Excelente, B: Bueno, R: Regular, M: Malo (criterio del agricultor). dda: días después de la aplicación.
\end{tabular}

Este tratamiento alcanzó valores de control entre el $91 \%$ y el $94 \%$, a los 35 días después de su aplicación, y superó a los demás tratamientos, en este período de evaluación (Tabla 34).

\section{Manejo Integrado de Arvenses (MIA)}

La filosofía del Manejo Integrado de Arvenses (MIA) es favorecer el predominio de arvenses de baja interferencia y de fácil manejo, y reducir las poblaciones de arvenses de alta interferencia; lo anterior para contribuir al establecimiento de coberturas y, por ende, a la conservación del suelo y el ambiente, sin afectar la productividad del cultivo y con los menores costos de producción. El MIA se basa en la integración conveniente y oportuna de los diferentes métodos de manejo de arvenses como son el método manual, el mecánico, el químico y el biológico.

Gómez (1990b) midió la erosión como el efecto de la desyerba de cafetales con azadón, machete y herbicidas bajo la modalidad de MIA. A partir del tercer año el cultivo se cerró y después de esta época se requirieron solamente parcheos esporádicos para controlar algunas arvenses. También se observaron pérdidas de suelo por erosión por debajo del nivel de tolerancia $(1,0$ t ha-año-1).

A partir de estas investigaciones sobre el MIA se obtuvieron las siguientes consideraciones: 
Tabla 34.

Intervalos promedio de control de arvenses (\%) en el cultivo del café con diferentes tratamientos (López et al., 2012).

\begin{tabular}{|c|c|c|c|c|c|}
\hline \multirow{3}{*}{ Tratamiento } & \multirow{3}{*}{ Dosis/ha } & \multicolumn{4}{|c|}{ Período después de la aplicación (días) } \\
\hline & & 15 & 21 & 28 & 35 \\
\hline & & \multicolumn{4}{|c|}{ Cobertura muerta (\%) } \\
\hline glufosinato de amonio $150 \mathrm{~g} \mathrm{~L}^{-1}$ & 1,5 & $81-88 A$ & $85-91 B$ & $86-93 A$ & $78-85 B$ \\
\hline glufosinato de amonio $150 \mathrm{~g} \mathrm{~L}^{-1}$ & 2,0 & $83-89 A$ & $85-90 B$ & $83-93 A$ & $79-86 B$ \\
\hline glifosato & 2,0 & $88-92 A$ & $86-90 \mathrm{~B}$ & $89-92 A$ & $91-94 \mathrm{~A}$ \\
\hline $\begin{array}{l}\text { glufosinato de amonio } 150 \mathrm{~g} \mathrm{~L}^{-1} \text { y } \\
\text { glifosato alternados en el tiempo }\end{array}$ & 2,0 & $78-92 A$ & $92-97 A$ & $91-96 A$ & $76-88 B$ \\
\hline
\end{tabular}

Intervalos de confianza promedio obtenidos a partir de 12 repeticiones por tratamiento, seis lecturas por repetición y tres aplicaciones en el tiempo. Valores con letras iguales no presentan diferencias estadísticas.

Para el desarrollo normal del cafeto, los dos primeros años son críticos desde el punto de vista del control de arvenses, así como para la erosión de los suelos debido a que se incurre en un control más frecuente de arvenses.

Cuando se realizan desyerbas selectivas en esta etapa del cultivo, las pérdidas de suelo por erosión se reducen entre el 95\% y el $97 \%$, debido a la presencia de las coberturas de baja interferencia.

Las arvenses nobles no deben invadir ni interferir con la zona de raíces del árbol (plato).

\section{Estrategias para el manejo de arvenses al nivel mundial}

Mortensen y Coble (1997) hacen un repaso general sobre las estrategias más importantes para el manejo de arvenses y analizan en ellas su factibilidad ambiental y económica, así:

Erradicación. Es la eliminación total de arvenses en el campo, convirtiéndose en una práctica costosa y benéfica sólo a corto plazo (en términos prácticos puede decirse que esta medida es casi imposible).

Profilaxis. Es una estrategia segura que incluye la aplicación de herbicidas preemergentes al suelo; este manejo puede causar detrimento de la calidad ambiental y desproteger los suelos, además que ocurre desperdicio de herbicidas y dinero. En café esta práctica puede ser útil en las etapas de almácigo y crecimiento vegetativo, en esta última, solamente en la zona de raíces del árbol (plato).

Remedial o de contención. Esta estrategia es usada para mantener la población de arvenses en un nivel específico bajo, tolerando la presencia de alguna población de arvenses en el cultivo, siempre y cuando las pérdidas en los rendimientos del cultivo sean iguales o menores que los costos de control, lo cual resulta en el manejo de arvenses basado en el conocimiento del umbral de las poblaciones de arvenses presentes. Las prácticas remediales son de gran valor potencial por presentar el menor costo y ser ambientalmente sanas; en este concepto se involucra el MIA investigado y recomendado por Cenicafé. 


\section{Establecimiento del Manejo Integrado de Arvenses (MIA)}

El manejo integrado de arvenses recomendado por Cenicafé, contempla los siguientes aspectos:

Plateo del cultivo. Esta labor debe realizarse manualmente en siembras nuevas y zocas de café hasta el primer año del cultivo; posteriormente puede hacerse mediante la aplicación de glifosato con el empleo del selector de arvenses. También puede realizarse en siembras nuevas y zocas de café con herbicidas preemergentes como el oxyfluorfen, lo cual brinda alta efectividad y persistencia en el control de arvenses de manera rentable.

Control manual. Esta práctica se realiza cuando en los cultivos se encuentren arvenses agresivas de difícil control por otros métodos, entre ellas: C. bonariensis (venadillo), Echinochloa sp. (arrocillo), Talinum paniculatum (verdolaga grande) y Colocasia esculenta (bore), arvenses enredaderas y parásitas, entre otras.

Control mecánico de arvenses. El control mecánico de las arvenses entre los surcos se realiza al tener en cuenta que, en los cultivos de café en etapa de levante, las arvenses no sobrepasen los $15 \mathrm{~cm}$ de altura y los $20 \mathrm{~cm}$ en cultivos en producción. Este control se realiza por medio de machete o guadañadora, al cortar las arvenses a una altura de 3 a $5 \mathrm{~cm}$ del suelo, sin dejar el suelo libre de coberturas.

Parcheos selectivos. Esta labor se realiza sobre las arvenses de interferencia alta y media, una vez estas alcanzan una altura aproximada de $15 \mathrm{~cm}$; para ello se utiliza el equipo selector de arvenses, al aplicar el herbicida glifosato (concentración comercial de $480 \mathrm{~g} \mathrm{~L}^{-1}$ de i.a.) a una concentración de la mezcla herbicida:agua del $10 \%$.
La integración de los anteriores sistemas de manejo promueve el establecimiento de las coberturas nobles a través del tiempo; cuando superan los $25 \mathrm{~cm}$ de altura deben cortarse a una altura de $5 \mathrm{~cm}$ aproximadamente.

\section{Descripción del selector de arvenses utilizado para realizar el MIA}

Según Marra y Carlson (1983), Mortensen y Coble (1997) y Higley y Pedigo (1997), el desarrollo de tecnologías que proporcionan alto grado de selectividad sobre las arvenses permite al agricultor realizar tratamientos remediales a sitios que excedan de manera económica los niveles de daño, por tal razón, la aplicación exitosa del MIA está ligada a la disponibilidad de esta tecnología.

Con el fin de facilitar el establecimiento de arvenses nobles o de interferencia baja y hacer uso racional y eficiente de herbicidas químicos, dentro de un manejo integrado de arvenses, Cenicafé desarrolló el selector de arvenses (Rivera, 1994), un equipo sencillo y liviano, diseñado para la aplicación de herbicidas sistémicos postemergentes, en forma selectiva, sobre las arvenses de alta interferencia.

En la Flgura 96 se describen las partes del selector para el manejo de arvenses (Rivera, 2000).

El equipo consiste de una te construida en tubería PVC o polipropileno, de 3/4" de diámetro interno, una altura de 1,30 m y ancho de $30 \mathrm{~cm}$ (Rivera, 2000).

\section{Volumen inicial de aplicación de herbicidas con el selector de arvenses}

Pruebas realizadas al variar el tamaño del equipo selector de arvenses determinaron 
que la velocidad de salida de la mezcla del herbicida es independiente del tipo de selector utilizado, debido a que esta depende directamente del volumen inicial de la aplicación, de la altura y del peso de la columna de la solución herbicida. En la Figura 97, se observa cómo a medida que disminuye la altura del líquido en el selector (volumen inicial de aplicación) disminuye también la velocidad de salida de la mezcla del herbicida. El equipo selector de arvenses expuesto en la Figura 97 con capacidad para $650 \mathrm{~cm}^{3}$, además de ser más liviano y cómodo para su manejo, es 54,3\% más eficiente en cuanto al ahorro de herbicida que el selector de arvenses de capacidad máxima de $1.200 \mathrm{~cm}^{3}$ (Salazar y Rivera, 2001).

\section{Concentración del herbicida en el selector de arvenses}

Con este equipo puede lograrse un control efectivo del $74 \%$ de arvenses de hoja ancha con la aplicación de glifosato a una concentración del 9\% (480 $\mathrm{g} \mathrm{L}^{-1}$ de i.a), y $87 \%$ de control de arvenses de hoja angosta a una concentración del 8\% (Figura 98), alcanzando para ambos casos una persistencia de control de 41 días (Figura 99).

\section{Efecto de la Iluvia sobre la aplicación}

Evaluaciones realizadas mediante la utilización de simulador de lluvias permitieron determinar que una lluvia de 60 $\mathrm{mm} \mathrm{h}^{-1}$ solo afecta el control si esta ocurre 30 minutos después de la aplicación del herbicida (Figura 100). Se encontró que una lluvia simulada de $60 \mathrm{~mm} \mathrm{~h}^{-1}$, ocurrida 30 minutos después de la aplicación del herbicida, difiere estadísticamente del tratamiento testigo (sin lluvia). El mismo aguacero ocurrido después de una hora no afecta significativamente la eficacia del control.

\section{Efecto del MIA sobre la producción del café}

En un experimento realizado en Chinchiná (Caldas) en siembras nuevas de café variedad Colombia, establecidas a $2 \times 1 \mathrm{~m}$, dos plantas por sitio (Salazar e Hincapié, 2009), la producción acumulada de café durante cuatro años, obtenida bajo el tratamiento MIA no presentó diferencias estadísticas con relación a la producción obtenida bajo el sistema de manejo de suelo libre de arvenses (Tabla 35). Bajo las mismas condiciones experimentales en la Variedad Castillo ${ }^{\circledR}$ se encontró el mismo comportamiento en la producción (Tabla 36). Es decir que el MIA además de evitar las pérdidas de suelo, no afecta la productividad del cultivo.

\section{Costos del manejo integrado de arvenses (MIA)}

Los costos siempre serán una inquietud y limitante para que el caficultor emprenda la adopción del MIA, al igual que las demás prácticas de conservación de suelos y aguas que se implementen, debe demostrarse la rentabilidad y efectividad en el tiempo.

Con el fin de evaluar las ventajas económicas del MIA, se compararon cinco sistemas de manejo de arvenses frecuentemente empleados por los caficultores (Tabla 37) con el manejo integrado de arvenses recomendado por Cenicafé; para ello, se seleccionaron cinco fincas cafeteras ubicadas en la zona cafetera central colombiana y en cada una de ellas se ubicaron dos parcelas, con un área que varió entre 0,25 y 0,50 ha cada una. En una parcela se llevó a cabo el manejo integrado de arvenses (MIA) y en la otra se realizó el manejo de arvenses que normalmente hace el agricultor (MT), consistente en manejo químico o mecánico 


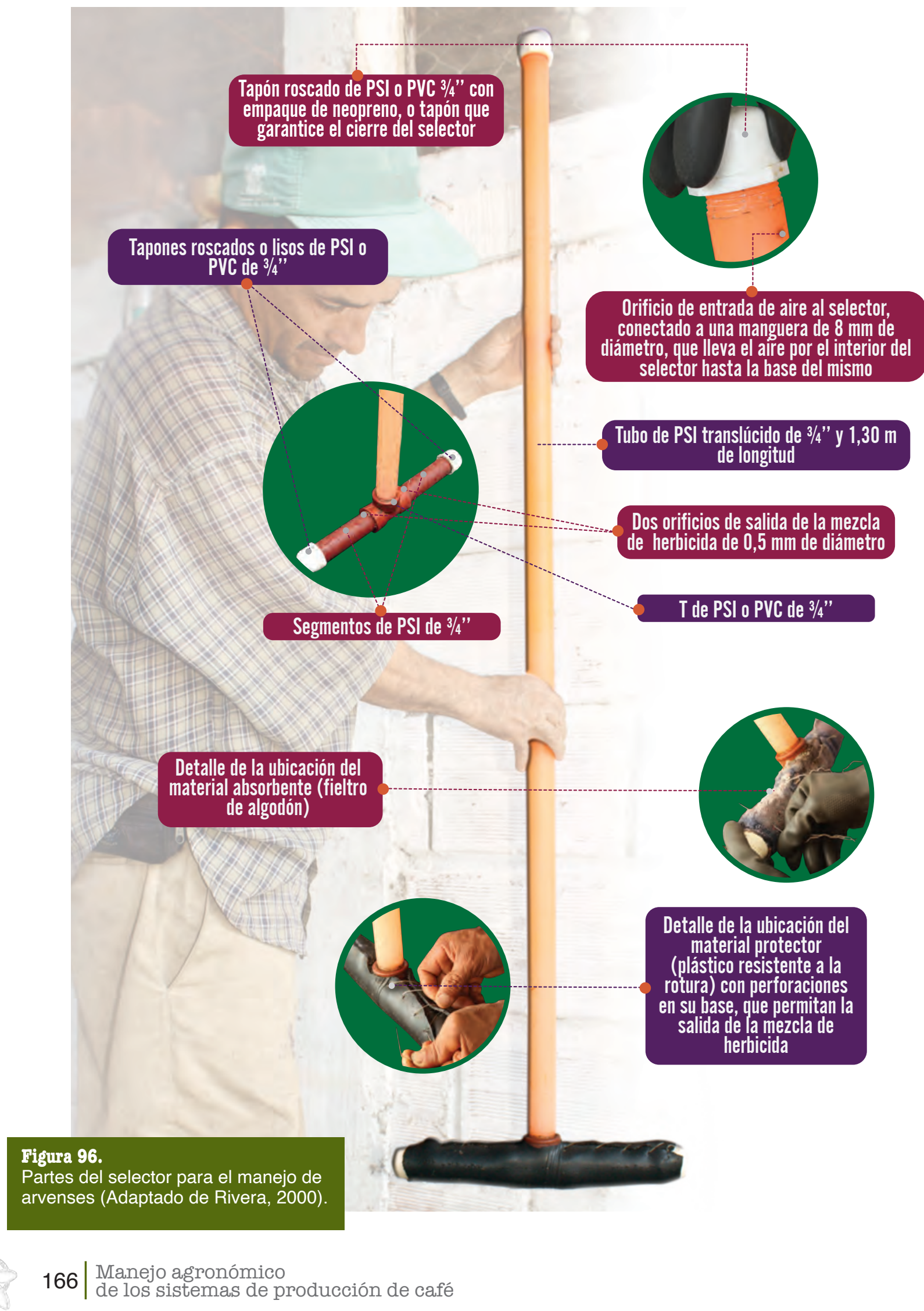



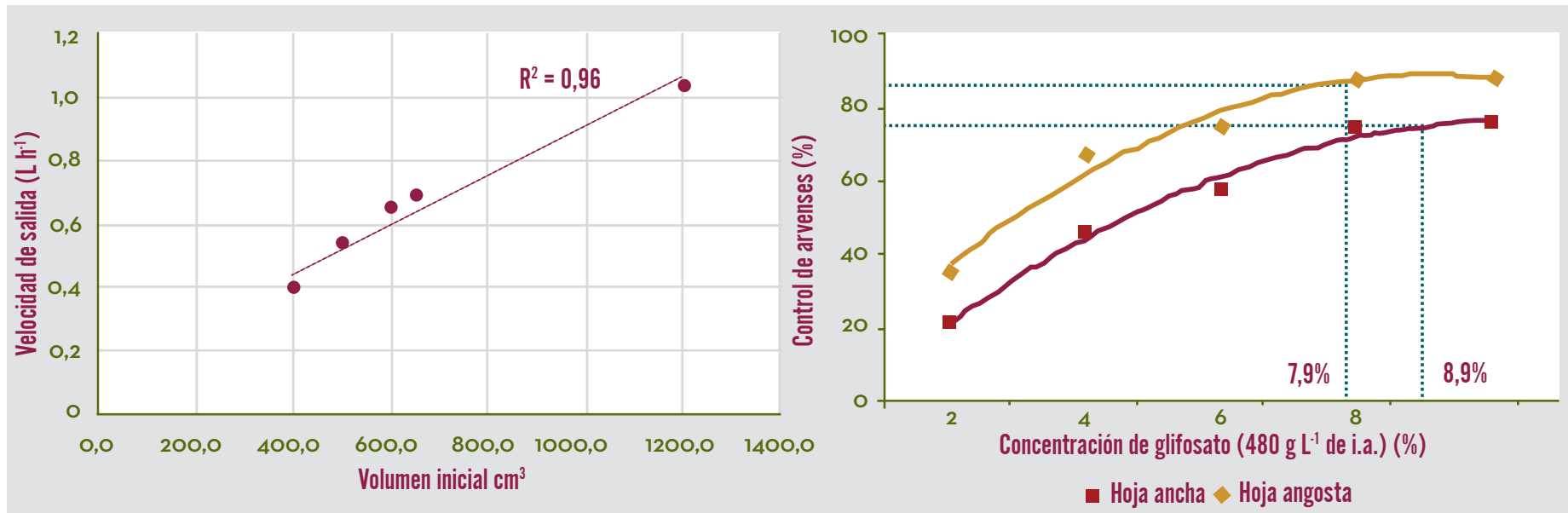

\section{Figura 97.}

Efecto del volumen inicial de aplicación sobre

la velocidad de salida de la mezcla herbicida (glifosato $480 \mathrm{~g} \mathrm{~L}^{-1}$ de i.a al 10\%).
Figura 98.

Efecto de diferentes concentraciones de glifosato aplicadas con el selector de arvenses sobre la eficacia del control.

dejando el suelo sin coberturas (Hincapié y Salazar, 2007).

El estudio se realizó durante dos años, y como variable respuesta se evaluaron los costos del manejo de ambos tratamientos. El manejo integrado de arvenses permitió la reducción de los costos a través del tiempo, comparado con el otro tipo de manejo. En el primer año estos disminuyeron en un $19,5 \%$ y en el segundo en un $47,0 \%$. En la Tabla 38 se observan jornales e insumos requeridos para cada método de manejo de arvenses en los diferentes sitios estudiados. Se observa como la mano de obra e insumos requeridos durante los primeros dos años del cultivo fueron menores para el manejo integrado de arvenses. Con el MIA se logró reducir el uso de herbicida en $29 \%$ durante el primer año y hasta el 63\% en el segundo año y también se logró la reducción en el uso del agua hasta el 95\%, al compararlo con el manejo tradicional de arvenses. En cuanto a la mano de obra las reducciones con el MIA son del $24 \%$ y $42 \%$ durante el primer y segundo año del cultivo, respectivamente.

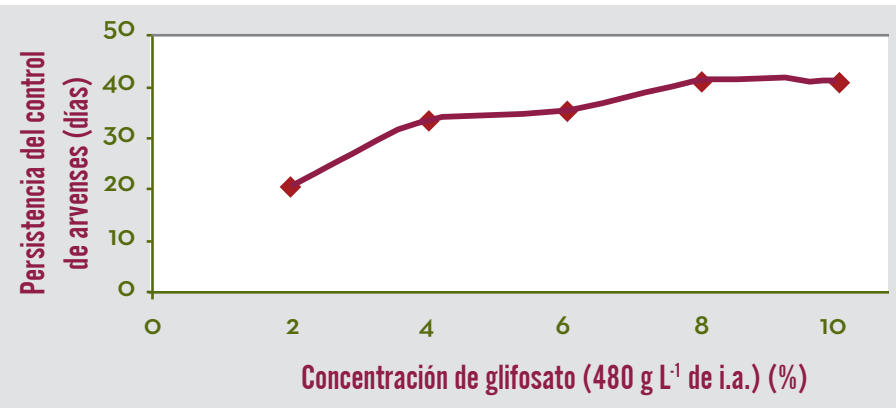

Figura 99.

Efecto de diferentes concentraciones de glifosato aplicadas con el selector de arvenses sobre la persistencia del control.

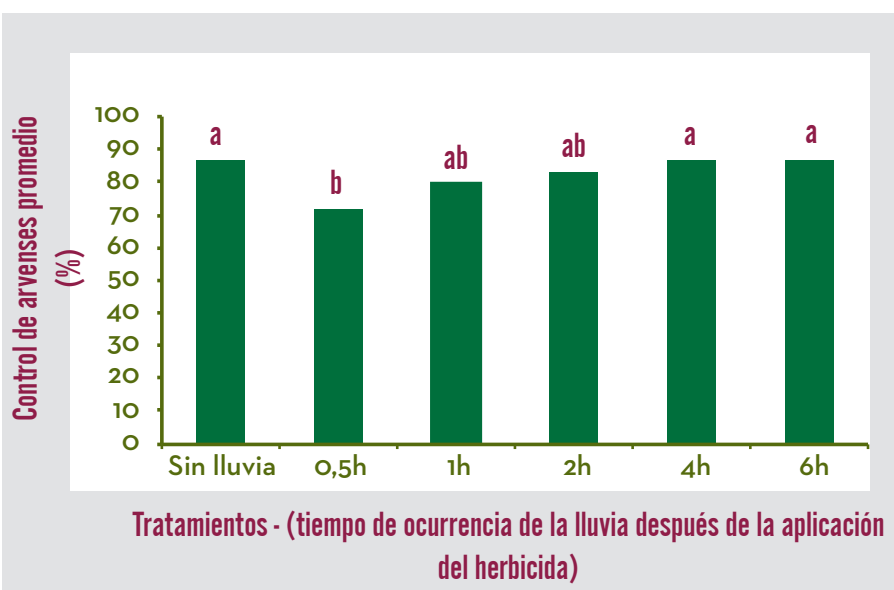

Figura 100.

Efecto de una lluvia simulada de $60 \mathrm{~mm} \mathrm{~h}^{-1}$ sobre el control de arvenses con selector (Tratamientos acompañados con letras iguales no difieren estadísticamente). 
Tabla 35.

Efecto del manejo integrado de arvenses (MIA) y el manejo del suelo libre de arvenses sobre la producción de café en la Estación Experimental Naranjal (Salazar e Hincapié, 2009).

\begin{tabular}{|c|c|c|c|c|c|}
\hline \multirow{2}{*}{ Tratamientos } & \multicolumn{5}{|c|}{ Producción de café pergamino seco (@ ha ${ }^{-1}$ ) } \\
\cline { 2 - 6 } & $\mathbf{1}^{\text {a }}$ Cosecha & $\mathbf{2}^{\text {a }}$ Cosecha & $\mathbf{3}^{\text {a }}$ Cosecha & $\mathbf{4}^{\text {a }}$ Cosecha & Acumulado \\
\hline MIA & $94,30 \mathrm{a}^{*}$ & $454,80 \mathrm{a}$ & $452,90 \mathrm{a}$ & $154,10 \mathrm{a}$ & $1156,1 \mathrm{a}$ \\
\hline Manejo libre de arvenses & $59,60 \mathrm{a}$ & $506,80 \mathrm{a}$ & $485,70 \mathrm{~b}$ & $185,70 \mathrm{a}$ & $1237,8 \mathrm{a}$ \\
\hline
\end{tabular}

*Valores seguidos de la misma letra son iguales estadísticamente.

Tabla 36.

Producción obtenida con diferentes tipos de cobertura en condiciones de la zona cafetera Central (Naranjal) (Salazar et al., 2012).

\begin{tabular}{|c|c|}
\hline Tratamiento & $\begin{array}{c}\text { Producción de café cereza (kg/parcela) } \\
\mathbf{2 0 0 7}-\mathbf{2 0 0 9}\end{array}$ \\
\hline MIA & $119,7 \mathrm{~A}^{*}$ \\
\hline Testigo libre de coberturas & $123,0 \mathrm{~A}$ \\
\hline
\end{tabular}

*Valores seguidos de la misma letra son iguales estadísticamente.

Tabla 3\%.

Manejo tradicional de arvenses que realizó el caficultor en cada una de las fincas estudiadas (Hincapié y Salazar, 2007).

\begin{tabular}{|c|c|}
\hline Localidad & Labores del manejo de arvenses hecho por agricultor \\
\hline A & $\begin{array}{l}\text { Un plateo manual inicial, luego control químico general con glifosato, aplicado con equipo } \\
\text { de aspersión y boquilla marcadora. }\end{array}$ \\
\hline B & $\begin{array}{l}\text { Plateo manual, control mecánico con machete dejando totalmente desnudo el suelo y } \\
\text { control químico general con glifosato aplicado con selector de arvenses. }\end{array}$ \\
\hline C & Control mecánico con guadaña dejando totalmente desnudo el suelo y plateo manual \\
\hline D & $\begin{array}{c}\text { Control mecánico con machete y químico general con glifosato aplicado con equipo de } \\
\text { aspersión. }\end{array}$ \\
\hline $\mathrm{E}$ & Control químico general con glifosato aplicado con equipo de aspersión. \\
\hline
\end{tabular}

En un año las labores del MIA fueron más frecuentes ( 7 a 11 veces) frente al manejo tradicional (cuatro a seis veces), lo que no se tradujo en mayores costos del MIA frente al manejo tradicional, ya que son más económicas y fáciles de realizar las desyerbas cuando son oportunas, además se reduce la ventaja competitiva de las arvenses frente al cultivo. Las desyerbas con machete o guadañadora que dejan el suelo sin coberturas, son los componentes más costosos dentro del MIA, en tanto el control con machete rápido, sin desnudar el suelo es un componente importante para el establecimiento de arvenses de fácil manejo y baja interferencia, el cual debe ser complementado con el control químico por parcheos o focos (Hincapié y Salazar, 2007). 
Tabla 38.

Jornales e insumos requeridos en cada tratamiento durante dos años.

\begin{tabular}{|c|c|c|c|c|c|}
\hline \multirow[b]{2}{*}{ Tratamiento } & \multirow[b]{2}{*}{ Año } & \multirow[b]{2}{*}{ Finca } & \multirow{2}{*}{$\begin{array}{l}\text { Mano de obra } \\
\text { Jornales/ha }\end{array}$} & \multicolumn{2}{|c|}{ Insumos } \\
\hline & & & & $\begin{array}{c}\text { Herbicida } \\
\text { L ha-1 }\end{array}$ & $\begin{array}{l}\text { Agua } \\
\text { L ha }\end{array}$ \\
\hline \multirow{10}{*}{ MIA } & \multirow{5}{*}{1} & A & 41,47 & 11,29 & 113 \\
\hline & & B & 38,32 & 9,59 & 96 \\
\hline & & C & 30,50 & 9,02 & 90 \\
\hline & & $\mathrm{D}$ & 36,60 & 11,55 & 115 \\
\hline & & $E$ & 25,80 & 9,33 & 93 \\
\hline & \multirow{5}{*}{2} & $A$ & 13,85 & 2,81 & 28 \\
\hline & & B & 25,68 & 3,17 & 32 \\
\hline & & C & 16,80 & 4,70 & 47 \\
\hline & & D & 19,08 & 4,00 & 40 \\
\hline & & $E$ & 17,28 & 4,30 & 43 \\
\hline \multirow{10}{*}{ MT } & \multirow{5}{*}{1} & A & 38,44 & 18,36 & 2.367 \\
\hline & & B & 51,56 & 7,68 & 155 \\
\hline & & C & 54,27 & - & - \\
\hline & & $D$ & 39,87 & 9,74 & 1.530 \\
\hline & & $E$ & 45,00 & 18,50 & 2.695 \\
\hline & \multirow{5}{*}{2} & A & 26,71 & 15,66 & 2.080 \\
\hline & & B & 40,71 & 5,44 & 70 \\
\hline & & C & 37,14 & 4,3 & 714 \\
\hline & & $D$ & 39,70 & - & - \\
\hline & & $E$ & 49,10 & 15,00 & 2.478 \\
\hline
\end{tabular}

MIA: Manejo integrado de arvenses, MT: Manejo del agricultor.

*Las fincas corresponden a las localidades descritas en la Tabla 37.

En cuanto a la cobertura del suelo ejercida por las arvenses, en 66 muestreos en lotes cultivados con café en las regiones cafeteras Norte, Centro y Sur de Colombia (Cenicafé, 2013), se encontró un valor de $47 \%(+/-20 \%)$ de cobertura promedio del suelo por las arvenses. Los mayores valores de cobertura del suelo por las arvenses se registraron en sitios donde el manejo y control de estas se hizo de manera integrado, con valores promedio del 59\%. La mayor diversidad de arvenses se alcanzó en cultivos donde se aplicó el MIA.

\section{Otras opciones de manejo de coberturas}

El maní forrajero (Arachis pintoi) y las coberturas nobles, ambos establecidos en platos y calles, no afectaron el desarrollo y la producción del café, en condiciones de Chinchiná (Caldas), cuando se incluyeron dentro un programa de manejo integrado de arvenses, mientras que Desmodium spp. afectó negativamente el desarrollo de la planta y la producción, especialmente cuando se estableció en los platos (Tabla 39) (Salazar et al., 2012). Una vez 
Tabla 39.

Efecto del manejo de coberturas en la producción de café cereza promedio obtenida en cultivo de café Variedad Castillo $^{\circledR}$ a libre exposición solar (Salazar et al., 2012).

\begin{tabular}{|c|c|}
\hline Tipo de manejo de cobertura & $\begin{array}{c}\text { Respuesta estadística } \\
\text { en la producción }\end{array}$ \\
\hline Plato con coberturas nobles vs. plato sin coberturas & $=$ \\
\hline Plato con coberturas nobles vs. plato con mulch & $=$ \\
\hline Plato sin coberturas vs. plato con mulch & $=$ \\
\hline Calle y plato sin coberturas vs. calle y plato con coberturas vivas & $=$ \\
\hline Calle y plato sin coberturas vs. plato con coberturas vivas & $=$ \\
\hline Calle y plato sin coberturas vs. MIA & $=$ \\
\hline Calle y plato sin coberturas vs. maní forrajero & $=$ \\
\hline Calle y plato sin coberturas vs. desmodium & ** \\
\hline
\end{tabular}

establecido el maní forrajero en el cultivo deben hacerse cortes cada 90 días y hacer control por parcheos sobre las arvenses agresivas.

\section{Eficiencia de herbicidas}

pre-emergentes en la etapa de levante del cultivo de café

En la etapa de levante del cultivo, el control de arvenses en la zona de raíces (plateo) es una práctica que demanda mano de obra de manera repetitiva, con el fin de garantizar el adecuado crecimiento de las plantas; esta actividad se realiza durante los primeros 12 o 18 meses de haber sido sembrada la planta de café en el campo. Es aquí donde la utilización de ingredientes activos pre-emergentes puede mantener el plato de la planta libre de arvenses durante un período de tiempo mayor al que transcurre con los controles manuales.

Por lo anterior, en las Estaciones Experimentales La Catalina en el municipio de Pereira (Risaralda) y Paraguaicito en el municipio de Buenavista (Quindío) se evaluaron cinco tratamientos para el control químico de arvenses en preemergencia, conformados por cinco ingredientes activos, el control manual (plateo) y un testigo sin algún tipo de control (Tabla 40). Por tratamiento se tuvieron ocho repeticiones, bajo el diseño de bloques completos al azar y mediante la prueba de Dunnett al 5\% se compararon los tratamientos con el testigo.

Los tratamientos conformados por los herbicidas se aplicaron en cada localidad una vez por semestre, durante un año. El área de aplicación y evaluación de los tratamientos estuvo constituida por la zona de raíces (plato) de la planta de café (Figura 101). Antes de iniciar la aplicación 
de los tratamientos se verificó que el área de raíces (platos) permaneciera sin la presencia de arvenses. Adicionalmente, se tuvo la precaución de que el suelo estuviera a capacidad de campo para favorecer la distribución y fijación de los herbicidas pre-emergentes en el área de aplicación. Para medir el control que ofrecieron los ingredientes activos en el campo se determinó que estos perdían efecto o residualidad cuando las arvenses emergidas en el área del plato alcanzaban un porcentaje de cobertura del $20 \%$ al $25 \%$, después de aplicar los productos.

Debido a las condiciones climáticas y de características del suelo, se encontraron diferencias del comportamiento de los tratamientos en ambas localidades. En condiciones de la Estación Paraguacito para la aplicación realizada en el primer semestre del año, la mayoría de los herbicidas habían perdido su efecto en el campo a los 90 dda (días después de la aplicación), al comportarse similar al testigo con un 35\% de cobertura de arvenses (Figura 102a). Para la aplicación realizada en el segundo semestre del año, a los 60 dda todos los ingredientes activos estudiados presentaron del $20 \%$ al $30 \%$ de cobertura de arvenses, demostrándose que en este período había culminado la residualidad del herbicida para el control de arvenses (Figura 102b).

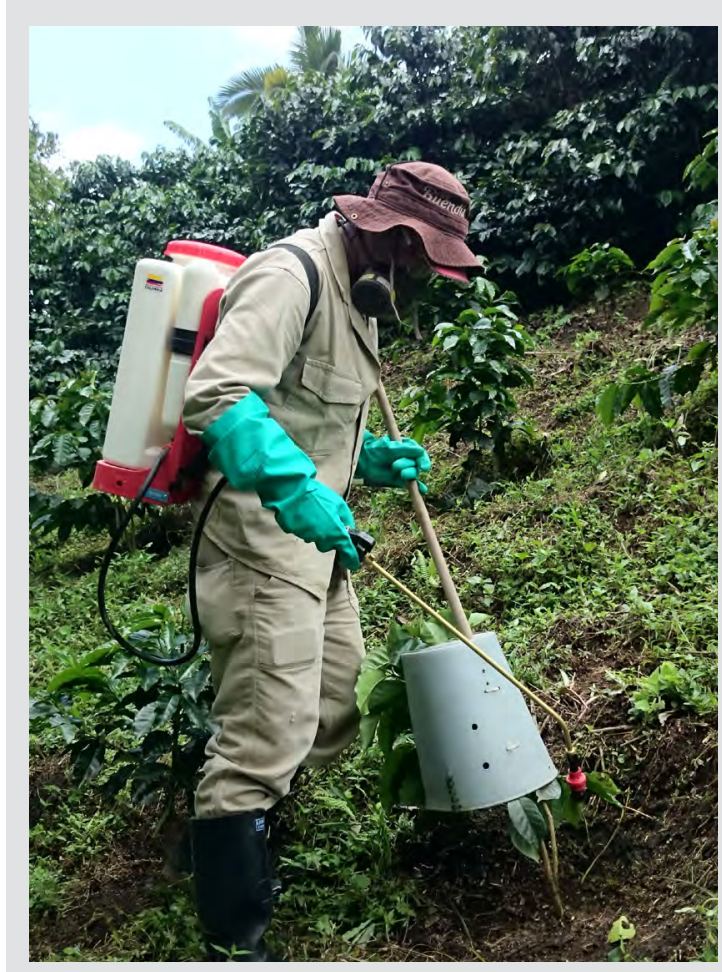

Figura 101.

Operario realizando la aplicación de los herbicidas preemergentes en la zona de raíces de la planta de café.

En la Estación La Catalina durante el primer semestre del año, los ingredientes activos oxyfluorfen, diurón y acetoclor ofrecieron un control de las arvenses en promedio del $20 \%$ de cobertura, a los 120 días después de su aplicación. Para esta misma época el testigo había alcanzado el $100 \%$ de cobertura de arvenses en

Tabla 40.

Descripción de los tratamientos.

\begin{tabular}{|c|c|c|c|c|}
\hline No. & Tratamiento & Nombre técnico & $\begin{array}{l}\text { Ingrediente activo } \\
\left(\mathbf{g ~ L}^{-1} \circ \mathbf{~ g} \cdot \mathbf{k g}^{-1}\right)\end{array}$ & $\begin{array}{l}\text { Dosis comercial } \\
\text { por hectárea }\end{array}$ \\
\hline 1 & Pre-emergente & oxyfluorfen & 240 & 3,0 L ha-1 \\
\hline 2 & Pre-emergente & diurón & 800 & $2,0 \mathrm{~kg} \mathrm{ha}^{-1}$ \\
\hline 3 & Pre-emergente & pendimetalina & 400 & $2,0 \mathrm{~L} \mathrm{ha}^{-1}$ \\
\hline 4 & Pre-emergente & acetoclor* & 600 & $2,5 \mathrm{~L} \mathrm{ha}^{-1}$ \\
\hline 5 & Pre-emergente & s-metolaclor* & 960 & $1,0 \mathrm{~L} \mathrm{ha}^{-1}$ \\
\hline 6 & Control manual & ----- & ----- & --- \\
\hline 7 & Testigo absoluto (sin control) & ----- & ----- & ----- \\
\hline
\end{tabular}

*Herbicidas sin registro ICA (Instituto Colombiano Agropecuario) para café, a abril de 2018. 
a.
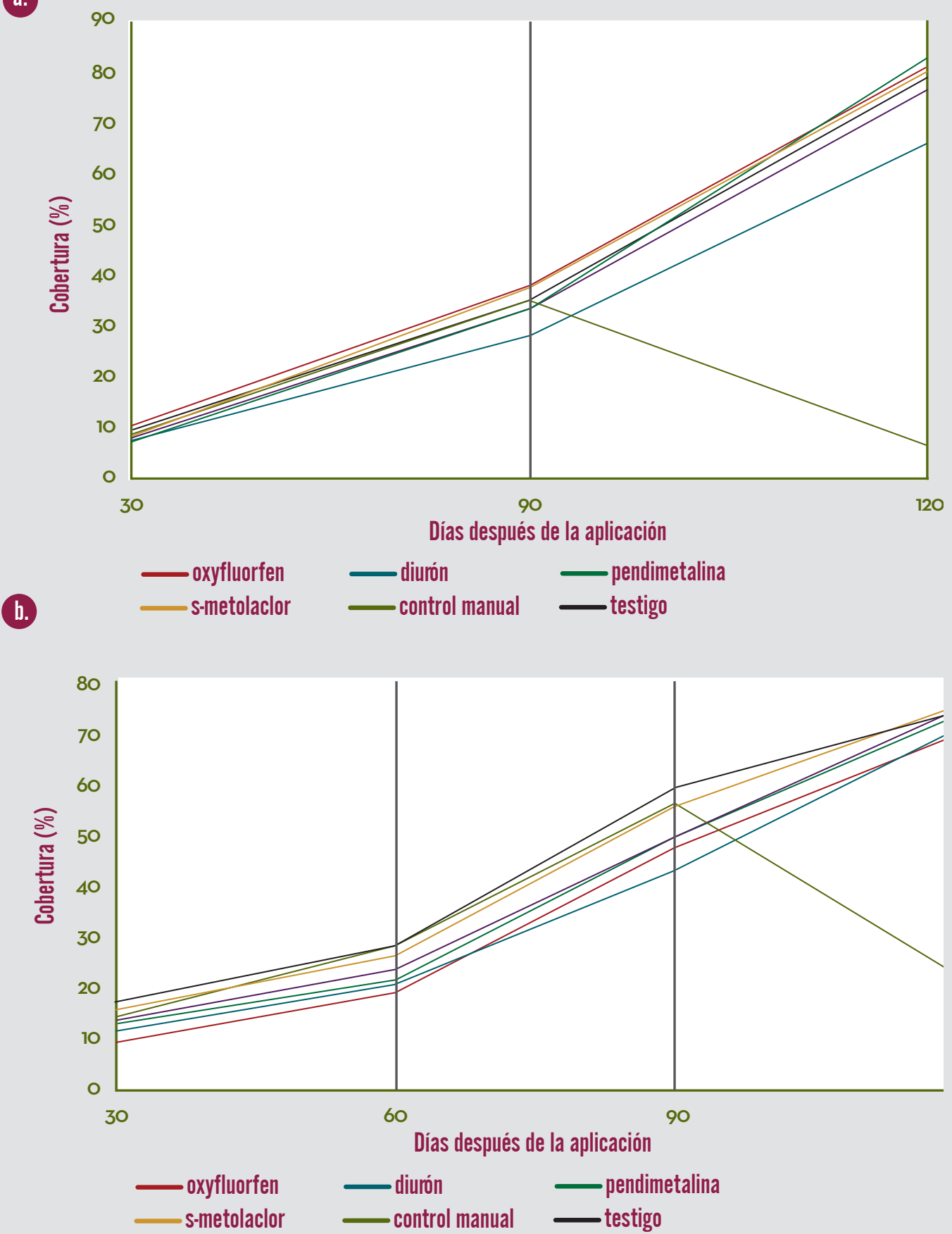

Figura 102.

Porcentajes de cobertura de arvenses en la zona de raíces (plato) del cultivo del café en respuesta a tratamientos de control en la Estación Experimental Paraguacito. a) Aplicación en el primer semestre del año. b) Aplicación en el segundo semestre del año. 
el plato (Figura 103a). En la aplicación realizada durante el segundo semestre los herbicidas oxyfluorfen, diurón, acetoclor y pendimentalina mostraron una cobertura del 20\% durante 140 días. S-metolaclor tuvo una persistencia menor que los demás herbicidas, pero demostró un control del $15 \%$ de cobertura de arvenses a los 120 días, mientras que el testigo obtuvo un $60 \%$ de cobertura a los 140 días (Figura 103b).

\section{Manejo de arvenses en almácigos de café}

Las arvenses interfieren con el cultivo del café en todas sus etapas de desarrollo, siendo la etapa del almácigo una de las más críticas, debido a que las arvenses pueden retrasar y afectar negativamente el crecimiento de la planta.

El manejo de arvenses en los almácigos de café puede realizarse al integrar principalmente los controles manual, cultural y químico. Las labores más recomendables son la desyerba manual y el manejo cultural, el cual consiste principalmente en la regulación de la luz para el almácigo, que se logra al reducirla al 50\% mediante el empleo de sombrío transitorio de especies arbustivas como leucaena, matarratón, higuerilla, crotalaria y guandul, entre otros, o al emplear elementos artificiales como polisombra, latas de guadua o residuos vegetales.

En el manejo cultural de arvenses juega un papel importante el manejo del sustrato previo al Ilenado de las bolsas, con el fin de controlar las semillas de las arvenses antes de iniciar su proceso de germinación, para ello es posible emplear la técnica de solarización del suelo que consiste en exponer el sustrato de suelo debajo de una cubierta plástica transparente, con espesor entre 40 a 100 micras y exponerlo

\section{Recomendación práctica}

Los herbicidas pre-emergentes al ser utilizados en el MIA ofrecen buena persistencia del control de arvenses en la zona de raíces del cultivo del café, los cuales según las condiciones de clima y suelo pueden oscilar entre 60 dda $y$ 140 dda, con niveles de cobertura de arvenses tolerables entre el $20 \%$ y el $25 \%$, lo cual contribuye

a la rentabilidad del cultivo.

*dda= días después de la aplicación

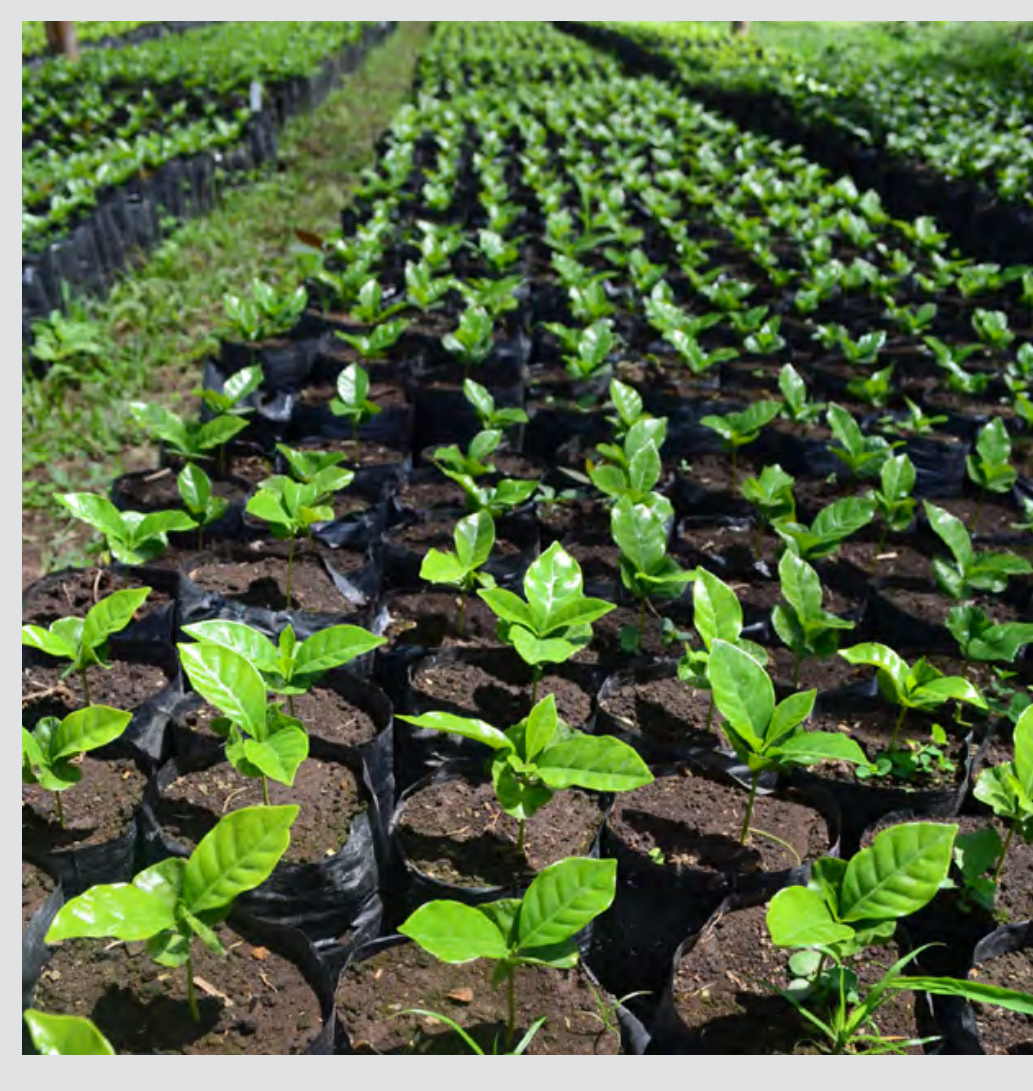

Manejo aǵronómico de los sistemas de producción de café 
a.
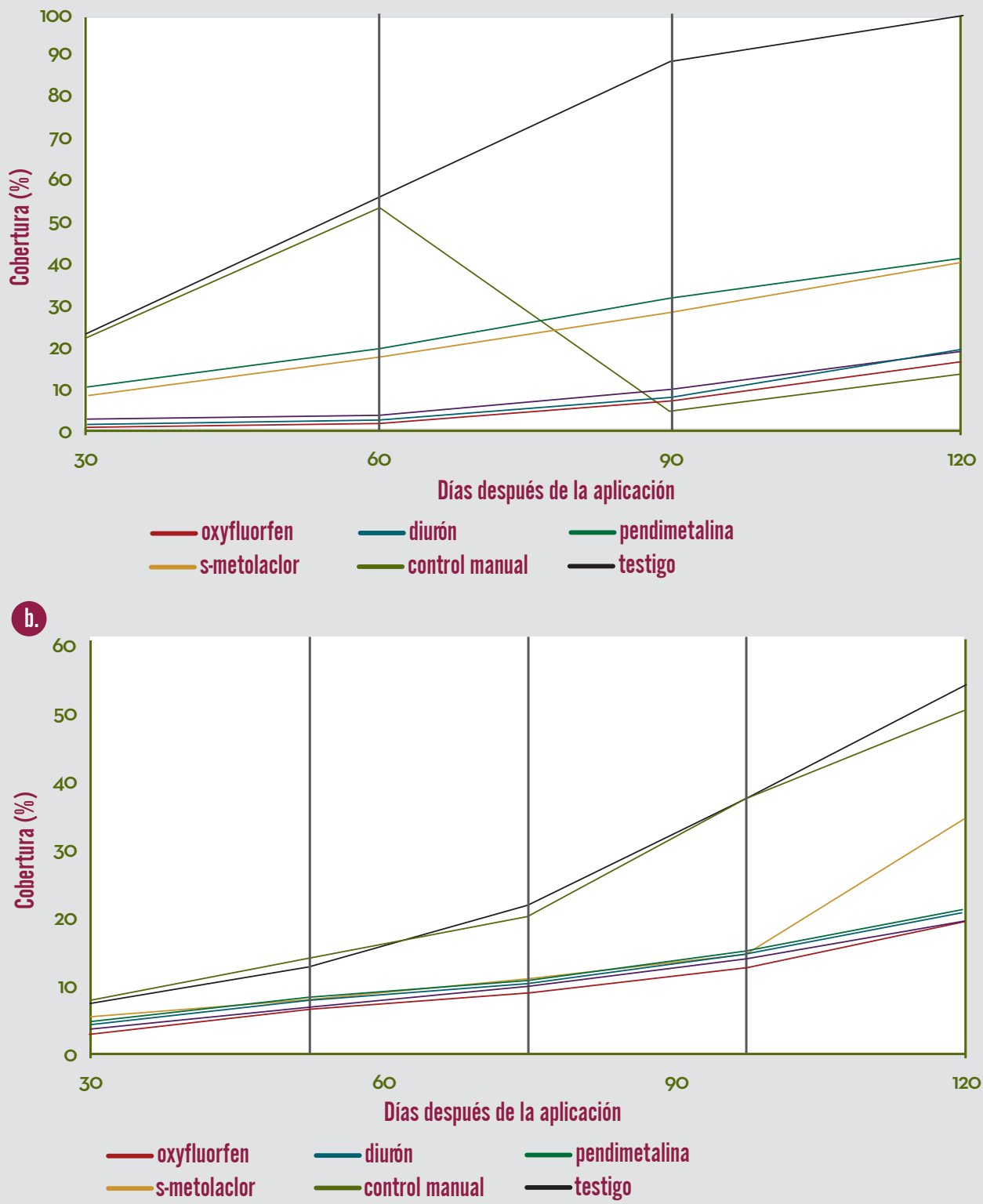

Figura 103.

Porcentajes de cobertura de arvenses en la zona de raíces (plato) del cultivo del café en respuesta a tratamientos de control en la Estación Experimental La Catalina. a) Aplicación en el primer semestre del año. b) Aplicación en el segundo semestre del año.

directamente a la acción del sol, durante cuatro a ocho semanas. Otra práctica para el control cultural de arvenses es el uso de cobertura muerta como cisco obtenido de la trilla de café o cascarilla de arroz sobre el suelo en el almácigo ya establecido, esta cobertura se utiliza como barrera física y retarda la germinación de las arvenses.

Para el control químico de arvenses en el almácigo, una vez llenadas las bolsas con 
el sustrato y antes de sembrar la planta de café, se recomienda por única vez aplicar el herbicida oxyflourfen, $240 \mathrm{~g}$ de ingrediente activo por litro de formulación en dosis de 1,0 a 2,0 L ha-1. La aplicación debe hacerse sobre el suelo sin arvenses y a capacidad de campo. Este herbicida actúa antes o en el momento de la germinación y sobre plántulas recién emergidas de las arvenses (2 a $4 \mathrm{~cm}$ de altura), controla gramíneas, cyperaceas y arvenses de hoja ancha y su persistencia de control alcanza hasta los 90 días. Su absorción por las raíces no es significativa por lo cual no hay problemas al trasplantar la planta de café a la bolsa después de su aplicación. Las investigaciones realizadas por Cenicafé indican que el oxyfluorfen es más eficaz que otros herbicidas pre-emergentes en el control de arvenses en almácigos, sin causar fitotoxicidad al café; sin embargo, debe evitarse el contacto del producto con los meristemos (Gómez et al., 1987). Este producto demanda de una estricta calibración del equipo de aplicación para no causar sobre-dosificación y con ello pérdidas por fitoxicidad.

\section{Criterios para el manejo de arvenses en el establecimiento de sistemas aǵroforestales con café}

En los sistemas agroforestales con café, debido al sombrío y el mulch generado por los árboles, existe menor incidencia de arvenses en especial de gramíneas, lo que permite una considerable reducción en las labores de desyerba. En la Figura 104a se observa la implementación del MIA en un sistema agroforestal del departamento de

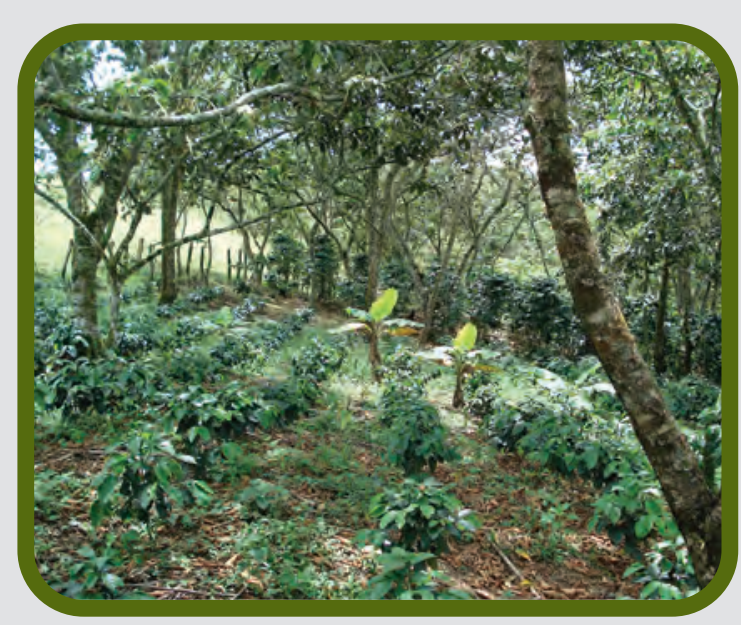

a.

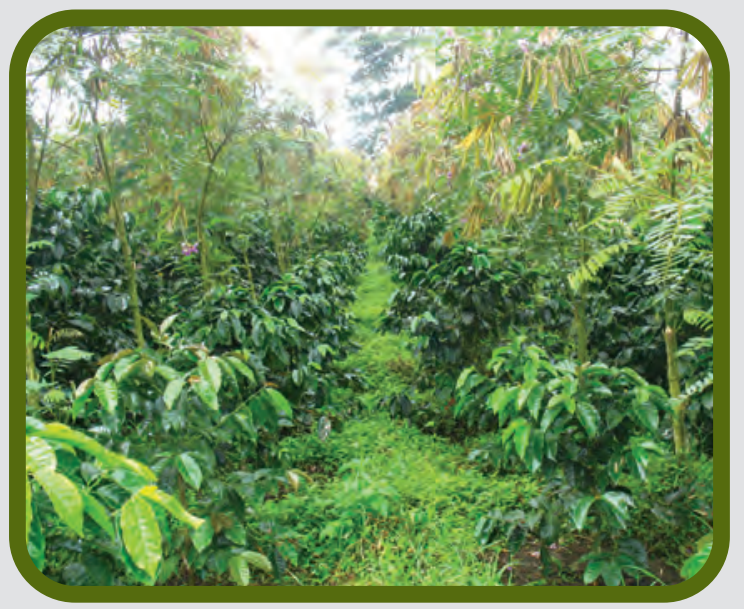

b.

Figura 104.

(a) Manejo integrado de arvenses en un sistema agroforestal con café.

Los Santos - Santander. (b). Manejo integrado de arvenses en un sistema

de café con sombrío transitorio de tefrosia en El Tambo - Cauca. 
Santander, Colombia y en la Figura 104b en un Sistema Agroforestal en El TamboCauca.

Durante el establecimiento tanto de los árboles como del cultivo debe evitarse que las arvenses compitan con ambos; debe hacerse el manejo de arvenses tanto al café como a las especies arbóreas. Por ello es necesario partir de un control de arvenses de alta interferencia, en especial, arvenses de hoja ancha de hábito trepador, plantas leñosas o semileñosas de raíz pivotante profunda, ya sea por medios manuales mecánicos o herbicidas químicos; el control manual-mecánico puede hacerse con machete o guadañadora, entre 3 a $5 \mathrm{~cm}$ del suelo, permitiendo que el suelo permanezca cubierto; si se hace con herbicidas este debe realizarse en forma selectiva por el método de parcheo, sobre los focos de las arvenses agresivas en especial gramíneas, helechos o arvenses de hoja ancha de hábito leñoso, semileñoso o trepador, permitiendo el establecimiento de arvenses nobles.

Los herbicidas utilizados pueden ser glifosato, paraquat, glufosinato de amonio o de tipo graminicida como fluazifop-p butil; en ningún caso se recomienda la aplicación de herbicidas pre-emergentes en forma generalizada, por los efectos que pueden tener sobre la erosión del suelo y debe tenerse mayor precaución en la aplicación de herbicidas como 2,4 $-D$, por la fitoxicidad que pueden generar en el café y los árboles. Los herbicidas deben aplicarse con equipos de aspersión - selectores de arvenses previamente calibrados, en el primer caso con una presión de aplicación entre 15 a 25 psi, con pantalla protectora antideriva y con un volumen de aplicación entre 200 a 300 L ha $^{-1}$ de agua.
Debe realizarse un plateo inicial (desyerba en la zona de raíces) del tamaño de la proyección del dosel de los árboles sobre el suelo. En café éste no debe ser inferior a los $30 \mathrm{~cm}$ de diámetro; el plateo puede realizarse manualmente evitando el uso de herramientas que disturben de alguna manera el sistema radicular del cultivo y los árboles, y en sistemas agroforestales con café más tecnificados, inmediatamente después del plateo manual, pueden aplicarse herbicidas pre-emergentes como el oxyfluorfen en las dosis recomendadas comercialmente para café, teniendo en cuenta las condiciones técnicas, principalmente de buena disponibilidad de humedad en el suelo.

Posterior al primer control de arvenses en calles y platos, los controles dependerán de la presión ejercida por las arvenses, la cual está determinada directamente por las condiciones de temperatura, radiación y humedad; en este sentido, deberán realizarse cortes con machete y/o guadañadora o parcheos sobre las arvenses agresivas con herbicidas. Durante el primer año se realizan entre seis y ocho parcheos y en casos con alta presión de arvenses, estos deben intercalarse con dos a tres cortes con machete o guadañadora que en ningún caso, tiendan a desnudar completamente el suelo. En el segundo año las labores y los costos disminuyen cerca del $50 \%$, dado el establecimiento de coberturas nobles y de los árboles. Al tercer año, las labores de manejo de arvenses se centran en los focos de mayor entrada de luz y al control de arvenses de hábito trepador y plantas parásitas, labores que deben hacerse en forma oportuna, evitando que alcancen un alto grado de desarrollo. El manejo de plantas parásitas debe realizarse conjuntamente con la labor de mantenimiento de los árboles y podas del 
café (deschupone). Después del tercer año, una vez haya disminuido la presión de gramíneas y se haya incrementado la presencia de arvenses de baja interferencia, deben realizarse podas de las arvenses con machete o guadañadora a 5,0 cm del suelo, cada 90 a 120 días.

Cuando el sistema se establece con cultivos transitorios intercalados o sombrío transitorio de especies leguminosas, debe hacerse el mismo manejo propuesto para el establecimiento del sistema agroforestal y las labores subsecuentes disminuyen dada la ocupación del espacio ofrecida por estas especies. Se requiere del repique de material vegetal en el suelo, una vez haya terminado su ciclo. En sistemas agroforestales de café se recomienda el establecimiento de coberturas leguminosas como maní forrajero.

\section{Manejo integrado de arvenses en sistemas de producción de café con cultivos intercalados}

Investigaciones realizadas por Moreno y Rivera (2003) han demostrado que es posible intercalar dos ciclos de producción de cultivos de maíz y fríjol con MIA sin que se afecte la producción del cultivo de café. En este sentido, Quiroz e Hincapié (2007) llevaron a cabo un experimento en la Estación Experimental Naranjal (Chinchiná, Caldas) en 24 predios de escorrentía, donde evaluaron ocho tratamientos con tres repeticiones, consistentes en cultivos transitorios de maíz (Zea mays), fríjol (Phaseolus vulgaris) y yuca (Manihot sculenta) intercalados entre las zocas de café, con y sin manejo integrado de arvenses (MIA). Las menores pérdidas de suelo se presentaron en el tratamiento café intercalado con maíz y MIA con un valor de $1,16 \mathrm{t} \mathrm{ha}^{-1}$, mientras que las mayores se presentaron en el tratamiento de café intercalado con fríjol y suelo desnudo, con un valor que superó las 4 t ha-1 (Tabla 41).

En los tratamientos de yuca intercalada con café, las pérdidas fueron similares a los demás tratamientos, pero estas se incrementaron después de la cosecha, superando a los demás tratamientos; en general, las pérdidas de suelo fueron menores en los tratamientos con MIA. Los resultados permitieron determinar que es posible reducir la erosión del suelo a niveles tolerables con el establecimiento de cultivos intercalados con el café, implementando el manejo integrado de arvenses. 
Para mejorar o mantener la productividad del cultivo del café es importante el correcto manejo de las poblaciones de arvenses de interferencia alta, al combinar de manera oportuna los diferentes métodos de control (manual, mecánico, químico, cultural y biológico). El manejo no debe basarse en solo un método de control, ya que pueden generarse problemas con especies de difícil manejo o inducir resistencia a herbicidas.

Las desyerbas tardías incrementan las poblaciones de las arvenses de interferencia alta, lo cual implica mayores costos de manejo a través del tiempo y efectos negativos en la producción. La aplicación de herbicidas químicos debe hacerse en forma racional y localizada sobre las arvenses de mayor interferencia al cultivo, preferiblemente en su estado vegetativo y mediante el uso del selector de arvenses, dentro de un plan de manejo integrado, al controlar el rebrote de los arvenses días después de un corte con machete o guadañadora. Lo anterior, con el fin de hacer más eficiente el control, facilitar la presencia de arvenses nobles, evitar toxicidad al cultivo y operarios y, principalmente, proteger los recursos naturales como el suelo, el agua y la biodiversidad.

Una forma de ser más rentable en la actividad cafetera es con la adopción del MIA, que apunta a mejorar la rentabilidad en cuatro formas:

1. Reducción de los costos de las desyerbas, Cenicafé ha demostrado en fincas cafeteras que este puede disminuir los costos entre el $20 \%$ para el primer año

y $45 \%$ en el segundo año de establecimiento del café, lo anterior debido a la reducción en la mano de obra e insumos necesarios para su realización.

2. Mantenimiento o mejoramiento de la productividad debido a controles más oportunos de arvenses.

3. Menor riesgo de aparición de arvenses resistentes a los herbicidas, que al escapar del control siguen compitiendo con el cultivo y además con su aparición se pierde la inversión y el esfuerzo hecho para su control.

4. Prevención de la erosión del suelo. Fstudios de Cenicafé han demostrado que cuando el suelo llega a un estado de erosión avanzado los rendimientos pueden disminuir hasta el 51\%, aun con la aplicación de fertilizantes.

Por lo anterior, es importante tener en cuenta la finalidad del MIA, que consiste en mantener el suelo con coberturas para conservarlo al igual que al agua, sin que se afecte la productividad y los costos de producción, lo cual se logra al disminuir la proporción de las poblaciones de arvenses agresivas y favorecer aquellas de más fácil manejo y mediana y baja interferencia con el cultivo o coberturas nobles. 
Tabla 41.

Pérdidas de suelo por erosión en sistemas de café con cultivos intercalados (Datos obtenidos durante diez meses de evaluación) (Quiroz e Hincapié, 2007).

\begin{tabular}{|c|c|c|c|}
\hline \multirow{2}{*}{ Manejo de arvenses } & Sistema de cultivo & \multicolumn{2}{|c|}{ Pérdidas de suelo (t ha-1) } \\
\cline { 2 - 4 } & Café//maíz & Media & 17,84 \\
\hline \multirow{3}{*}{ Sin MIA } & Café//fríjol & $3,35 \mathrm{~b}$ & 31,50 \\
\hline \multirow{3}{*}{ Con MIA } & Café//yuca & $4,82 \mathrm{a}$ & 6,56 \\
\hline & Café & $3,28 \mathrm{~b}$ & 24,39 \\
\hline & Café//maíz & $3,51 \mathrm{ab}$ & 17,08 \\
\hline & Café//fríjol & $1,57 \mathrm{c}$ & 41,03 \\
\hline & Café//yuca & $3,46 \mathrm{ab}$ & 36,72 \\
\hline & Café & $2,81 \mathrm{ab}$ & 13,03 \\
\hline
\end{tabular}

Letras distintas entre tratamientos indican diferencias significativas según prueba DMS al 5\% (Diferencia mínima significativa = 1,4092). 


\section{Literatura citada}

Aldrich, R. J. (1987). Predicting Crop Yield Reductions from Weeds. Weed Technology, 1(3), 199-206. https://www.jstor.org/stable/3987469

Alvarado, G., \& Moreno-Ruiz, G. (1999). ¿Cómo se distribuye anualmente la cosecha de las variedades Caturra y Colombia? Avances Técnicos Cenicafé, 260, 1-4. http://hdl.handle.net/10778/4172

Alvarado, G., Posada, H. E., \& Cortina, H. A. (2005). Castillo: Nueva variedad de café con resistencia a la roya. Avances Técnicos Cenicafé, 337, 1-8. http://hdl.handle.net/10778/401

Alvarado, G., Posada, H. E., Cortina, H. A., Duque-Orrego, H., Baldión, J. V., \& Guzmán-Martínez, O. (2005a). La variedad Castillo Naranjal para regiones cafeteras de Caldas, Quindío, Risaralda y Valle. Avances Técnicos Cenicafé, 338, 1-8. http://hdl.handle.net/10778/413

Alvarado, G., Posada, H. E., Cortina, H. A., Duque-Orrego, H., Baldión, J. V., \& Guzmán-Martínez, O. (2005b). La variedad Castillo Paraguaicito para regiones cafeteras de Quindío y Valle del Cauca. Avances Técnicos Cenicafé, 339, 1-8. http://hdl.handle.net/10778/417

Alvarado, G., Posada, H. E., Cortina, H. A., Duque-Orrego, H., Baldión, J. V., \& Guzmán-Martínez, O. (2005c). La variedad Castillo El Rosario para regiones cafeteras de Antioquia, Risaralda y Caldas. Avances Técnicos Cenicafé, 340, 1-8. http://hdl.handle.net/10778/403

Alvarado, G., Posada, H. E., Cortina, H. A., Duque-Orrego, H., Baldión, J. V., \& Guzmán-Martínez, O. (2005d). La variedad Castillo Pueblo Bello para las regiones de Magdalena, Cesar La Guajira y Norte de Santander. Avances Técnicos Cenicafé, 341, 1-8. http://hdl.handle.net/10778/342

Alvarado, G., Posada, H. E., Cortina, H. A., Duque-Orrego, H., Baldión, J. V., \& Guzmán-Martínez, O. (2005e). La variedad Castillo Santa Bárbara para las regiones cafeteras de Cundinamarca y Boyacá. Avances Técnicos Cenicafé, 342, 1-8. http://hdl.handle.net/10778/383

Alvarado, G., Posada, H. E., Cortina, H. A., Duque-Orrego, H., Baldión, J. V., \& Guzmán-Martínez, O. (2006). La variedad Castillo La Trinidad para regiones cafeteras de Tolima. Avances Técnicos Cenicafé, 343, 1-8. http://hdl.handle.net/10778/348

Arcila, A. (2011). La floración, indicador del ataque de la broca. Brocarta, 44, 1-2. https://www.cenicafe. org/es/publications/brc044.pdf

Arcila, J., Farfán, F., Moreno, A. M., Salazar, L. F., \& Hincapié, E. (2007). Sistemas de producción de café en Colombia. Cenicafé. http://hdl.handle.net/10778/720 
Arcila, J., Buhr, L., Bleiholder, H., Hack, H., \& Wicke, H. (2001). Aplicación de la escala BBCH ampliada para la descripción de las fases fenológicas del desarrollo de la planta de café Coffea sp. Boletín Técnico Cenicafé, 23, 1-32. http://hdl.handle.net/10778/578

Arcila, J., \& Farfán, F. F. (2007). Consideraciones sobre la nutrición mineral y orgánica en los sistemas de producción de café. En J. Arcila, F.F. Farfán, A. M. Moreno, L.F. Salazar, \& E. Hincapié (Eds.), Sistemas de producción de café en Colombia (pp. 201-232). Cenicafé. http://hdl.handle.net/10778/720

Arcila, J., Jaramillo-Robledo, A., Baldión, J. V., \& Bustillo-Pardey, A. E. (1993). La floración del cafeto y su relación con el control de la broca. Avances Técnicos Cenicafé, 193, 1-6. http://hdl.handle.net/10778/348

Aristizábal, L. F., Salazar, H. M., \& Mejía, C. G. (2002). Evaluación de dos componentes del manejo de la broca en la renovación de cafetales mediante investigación participativa. Avances Técnicos Cenicafé, 295, 1-8. http://hdl.handle.net/10778/1048

Basel, E. H., \& Berlin, S. H. (1980). Panicoid grass weeds: Grass weeds 1. CIBA-GEYGY Ltd.

Beer, J., Harvey, C. A., Ibrahim, M., Harmand, J. M., Somarriba, E., \& Jiménez, F. (2003, September 2128). Service functions of agroforestry systems. XII World Forestry Congress, Québec, Canada. http://hdl. handle.net/11554/8365

Beer, J.W., Muschler, R.G., Kass, D., \& Somarriba, E. (1998). Shade management in coffee and cacao plantations. Agroforestry Systems, 38, 139-164. https://doi.org/10.1023/A:1005956528316

Behling, A., Sanquetta, C. R., Dalla, A. P., Caron, B., Simon, A., Behling, M., \& Schmidt, D. (2015). Conversion efficiency of photosynthetically active radiation intercepted in biomass in stands of black wattle in Brazil. Bosque, 36(1), 61-69. http://dx.doi.org/10.4067/S0717-92002015000100007

Benavides, P. (2013). Aciertos y desaciertos en las prácticas de renovación de cafetales infestados por broca. Brocarta, 48, 1-2. https://www.cenicafe.org/es/publications/brc048.pdf

Blanco, H.G., Oliveira, D. A. \& Puppo, E. I. H. (1978, outubro). Efeitos da epoca de controle do mato sobre a producao de uma lavoura de café em formacao: Resultados de 3 años de observacoes. VI Congresso Brasileiro de Pesquisas Cafeeiras, Sao Paulo, Brasil.

Bradshaw, L., \& Rice, K. L. (1998). Competencia por agua entre el café y tres coberturas vivas (Arachis, Desmodium y malezas) en Nicaragua. Agronomía Costarricense, 21(1), 51-60. https://www.mag.go.cr/ rev agr/v22n01 051.pdf

Braverman, M. P. (1998). Simulated glufosinate drift on rice and soybean. Proceeding Southern Weed Science Society, 51, 269.

Buechel, T. (2017, septiembre 12). Cómo medir la calidad y la cantidad de luz en el invernadero. https:/l www.pthorticulture.com/es/centro-de-formación/cómo-medir-la-calidad-y-la-cantidad-de-luzl 
Bustillo, A. E. (2007). El manejo de cafetales y su relación con el control de la broca del café en Colombia. Boletín Técnico Cenicafé, 24, 1-40. http://hdl.handle.net/10778/579

Camayo, G. C., \& Arcila, J. (1997). Desarrollo floral del cafeto en condiciones de zona cafetera colombiana (Chinchiná-Caldas). Avances Técnicos Cenicafé, 245, 1-8. http://hdl.handle.net/10778/4174

Castillo, J. (1975). Producción y características de germoplasma de café introducido a Colombia. Revista Cenicafé, 26(1), 3-26. http://hdl.handle.net/10778/4194

Castro, A. M., \& Rivillas, C. A. (2003). Manejo sostenible de la llaga macana en cafetales renovados por zoca. Avances Técnicos Cenicafé, 312, 1-8. http://hdl.handle.net/10778/4175

Castro, A. M., Rivillas-Osorio, C. A., Serna-Giraldo, C., \& Mejía, C. G. (2008). Germinadores de café: construcción, manejo de Rhizoctonia solani y costos. Avances Técnicos Cenicafé, 368, 1-12. http://hdl. handle.net/10778/4176

Centro Nacional de Investigaciones de Café. (2001). Informe Anual Cenicafé 2001 (p. 37-38). https://doi. org/10.38141/10783/2001

Centro Nacional de Investigaciones de Café. (2013). Informe Anual Cenicafé 2013. https://doi. org/10.38141/10783/2013

Centro Nacional de Investigaciones de Café. (2010). Anuario meteorológico cafetero 2009. Cenicafé. https:/l biblioteca.cenicafe.org/bitstream/10778/660/1/anu2009.pdf

Centro Nacional de Investigaciones de Café. (2016). Anuario meteorológico cafetero 2014. Cenicafé. https:/l biblioteca.cenicafe.org/bitstream/10778/660/6/anu2014.pdf

Centro Nacional de Investigaciones de Café. (2017a). Anuario meteorológico cafetero 2016. Cenicafé. https://biblioteca.cenicafe.org/bitstream/10778/660/14/anu2016.pdf

Centro Nacional de Investigaciones de Café. (2017b). Informe Anual Cenicafé 2017 (p. 83). https://doi. org/10.38141/10783/2017

Centro Nacional de Investigaciones de Café. (2018). Guía: Más agronomía, más productividad. Cenicafé. https://www.cenicafe.org/es/publications/GuiaMasAgronomia.pdf

Chávez-Pascual, E. Y., Rodríguez-Ortiz, G., Carrillo-Rodríguez, J. C., Enríquez, J. R., Chávez-Servia, J. L., \& Campos-Ángeles, G. V. (2013). Aboveground biomass expansion factors for Pinus chiapensis (Mart.) Andresen. Revista Mexicana de Ciencias Agrícolas, 4(6), 1273-1284.

Chee, Y. K., Alif, A. F., \& Chung, G. F. (1992). Management of weeds in plantation crops in 2000. En A. Aziz, S.A. Kadir, \& H.S (Eds.), Barlow Pest management and the environment in 2000. (pp. 270-280). CAB International.

$182 \mid \begin{aligned} & \text { Manejo agronómico } \\ & \text { de los sistemas de producción de café }\end{aligned}$ 
Coble, H., \& Mortensen, D. (1992). The threshold concept and its application to weed science. Weed Science, 6(1), 191-195. https://doi.org/10.1017/S0890037X00034552

Cordero, J., \& Boshier, D. H. (2003). Árboles de Centroamérica: un manual para extensionistas. CATIE. http://orton.catie.ac.cr/repdoc/a11445e/a11445e.pdf

Cousens, R. (1985). A simple model relating yield loss to weed density. Annals of Applied Biology, 107(2), 239-252. https://doi.org/10.1111/j.1744-7348.1985.tb01567.x

Cruz, R (1989). Las malezas en el cultivo del fríjol en América Latina: Guía de estudio como complemento de la unidad audio tutorial sobre el mismo tema. CIAT.

Damatta, F.M., Ronchi, C. P., Maestri, M., \& Barros, R. S. (2007). Ecophysiology of coffee growth and production. Brazilian Journal of Plant Physiology, 19(4), 485-510. https://doi.org/10.1590/S167704202007000400014

Dew, D. A. (1972). An index of competition for estimating crop loss due to weeds. Canadian Journal Research, 52(6), 921-927. https://www.nrcresearchpress.com/doi/pdf/10.4141/cjps72-159.

Díaz, E., Loeza, J. M., Campos, J. M., Morales, E. J., Domínguez, A., \& Franco, O. (2013). Eficiencia en el uso de la radiación, tasa de asimilación neta e integral térmica en función del fósforo en maíz (Zea mays L.). Agrociencia, 47(2), 135-146.

Doll, J. (1981). Factores que condicionan la eficacia de los herbicidas. CIAT.

Doll, J. (1982). Los herbicidas: Modo de actuar y síntomas de toxicidad. CIAT.

Duarte, O., Ríos, G., \& Silva, J. (1996). Conceptos básicos sobre la metodología de sistemas de producción. En Instituto Colombiano Agropecuario (Ed.), Módulos para la capacitación metodológica de las UMATA (pp. 1-16). ICA.

Duicela, L. A., \& Corral, R. (2003). Influencia de las podas y regulación de sombra sobre la producción de cafetales arábigos. COFENAC.

Duke, S. O. (2012). Why have no new herbicide modes of action appeared in recent years?. Pest Management Science, 68(4), 505-512. https://doi.org/10.1002/ps.2333

Duque, H. (2001). Análisis económico de doce prácticas para mejorar el desempeño de las fincas cafeteras. Cenicafé.

Duque, H. (2004). Cómo reducir los costos de producción en la finca cafetera. Cenicafé. 
Enríquez, G., \& Martínez, A. (1984). La sombra para el cacao: Revisión de literatura y bibliografía anotada. CATIE. http://hdl.handle.net/11554/2720

Escobar, G., \& Berdegué, J. (1990). Tipificación de sistemas de producción agrícola. RIMISP. http://hdl. handle.net/10625/3969

Eshetu, T. (2001, May 14-18). Weed flora and weed control practices in coffee (Coffea arabica L.) in Ethiopia: A review. Proceedings of 19th International Scientific Colloquium on Coffee Trieste, Italy. https://www.asiccafe.org/conference/19th-international-scientific-colloquium-coffee/weed-flora-and-weed-control-practices

Farfán, F. F. (2007). Producción de café en sistemas agroforestales. En J. Arcila, F.F. Farfán, A. M. Moreno, L.F. Salazar, \& E. Hincapié (Eds.), Sistemas de producción de café en Colombia (pp. 161-200). Cenicafé. http://hdl.handle.net/10778/720

Farfán, F. F. (2014). Agroforestería y sistemas agroforestales con café. Cenicafé. https://www.cenicafe.org/ es/publications/AgroforesteriaSistemasCafe.pdf

Farfán, F., \& Baute, J. E. (2009). Efecto del arreglo espacial del café y del sombrío sobre la producción de café. Revista Cenicafé, 60(4), 313-323. http://hdl.handle.net/10778/68

Farfán, F., Bermúdez, L. N., \& González, N. E. (2002). Evaluación de herramientas para valorar el porcentaje de sombras en sistemas agroforestales con café. Avances Técnicos Cenicafé, 472, 1-8. http://hdl.handle. net/10778/4177

Farfán, F., \& Jaramillo, A. (2008). Efecto de la cobertura vegetal muerta y arbórea sobre la disponibilidad de agua en el suelo en sistemas agroforestales con café. Revista Cenicafé, 59(1), 39-54. http://hdl. handle. net/10778/162

Farfán, F., \& Jaramillo-Robledo, A. (2009). Sombrío para el cultivo del café según la nubosidad de la región. Avances Técnicos Cenicafé, 379, 1-8. http://hdl.handle.net/10778/376

Farfán, F., \& Mestre, A. (2004). Respuesta del café cultivado en un sistema agroforestal a la aplicación de fertilizantes. Revista Cenicafé, 55(2), 161-174. http://hdl.handle.net/10778/168

Farfán, F., Rendón, J. R., \& Menza, H. D. (2016). Densidad de siembra de Coffea arabica variedad Tabi en sistemas agroforestales en tres zonas cafeteras de Colombia. Revista Cenicafé, 67(2), 52-57. http://hdl. handle.net/10778/728

Farfán, F., \& Sánchez, P. M. (2016). Densidad de siembra del café variedad Castillo® en sistemas agroforestales, en el departamento de Santander-Colombia. Cenicafé, Revista Cenicafé, 67(1), 55-62. http://hdl.handle.net/10778/678 
Farfán, F., \& Solarte, C. R. (2008). Efecto de la cobertura arbórea y vegetal muerta sobre la producción de café, en una localidad de la zona cafetera sur de Colombia. Revista Cenicafé, 59(1), 155-164. http://hdl. handle.net/10778/206

Farfán, F., \& Urrego, B. (2004). Comportamiento de las especies forestales Cordia alliodora, Pinus oocarpa y Eucalyptus grandis como sombrío e influencia en la productividad del café. Revista Cenicafé, 55(4), 317329. http://hdl.handle.net/10778/243

Fassbender, H. W. (1993). Modelos edafológicos de sistemas agroforestales. CATIE. http://orton.catie. ac.cr/repdoc/A0813e/A0813e.pdf

Federación Nacional de Cafeteros de Colombia. (1993). Manual de uso de fotografías aéreas: Aplicación al sistema de información cafetera. FNC.

Flórez, C. P., Maldonado, C. E., Cortina, H. A., Moncada, M. P., Montoya, E. C., Ibarra, L. N., Unigarro, C. A., Rendón-Sáenz, J., \& Duque-Orrego, H. (2016). Cenicafé 1: Nueva variedad de porte bajo, altamente productiva, resistente a la roya y al CBD, con mayor calidad física del grano. Avances Técnicos Cenicafé, 469, 1-8. http://hdl.handle.net/10778/4178

Flumignan, D. L., Teixeira, R., \& Cavenaghi, C. E. (2011). Evapotranspiration components and dual crop coefficients of coffee trees during crop production. Agricultural Water Management, 98(5), 791-800. https:/l doi.org/10.1016/j.agwat.2010.12.002

Friessleben, U., Pohlan, J., \& Franke, G. (1991). The response of Coffea arabica L. to weed competition. Café Cacao Thé, 35(1), 15-20.

Fuentes, C. (1986). Metodología y técnicas para evaluar las poblaciones de malezas y su efecto en los cultivos. Revista Comalfi, 13, 29-50.

Fuentes, C. L., Almario, O., \& Cifuentes, F. (1999). Malezas cyperáceas asociadas con el cultivo del arroz en Colombia. AGREVO.

Gaitán, A., Villegas, C., Rivillas-Osorio, C. A., Hincapié, E., \& Arcila, J. (2011). Almácigos de café: Calidad fitosanitaria manejo y siembra en el campo. Avances Técnicos Cenicafé, 404, 1-8. http://hdl.handle. net/10778/350

Galvis, C. A. (2002). El mal rosado del cafeto. Avances Técnicos Cenicafé, 299, 1-8. http://hdl.handle. net/10778/843

Galvis, C. A., \& Salazar, L. F. (2009). Identifique y prevenga los daños en cafetales por herbicidas. Avances Técnicos Cenicafé, 383, 1-12. http://hdl.handle.net/10778/356

Gil, Z., Constantino, L. M., Martínez, H., \& Benavides Machado, P. (2013). Aprenda a manejar la arañita roja del café. Avances Técnicos Cenicafé, 436, 1-8. http://hdl.handle.net/10778/473 
Gil, L. F. (2001). Descripción de daños ocasionados por Colletotrichum sp. en flores y frutos de café en Colombia. Avances Técnicos Cenicafé, 288, 1-4. http://hdl.handle.net/10778/4179

Gil, L. F., \& Leguizamón, J. E. (2000). La muerte descendente del cafeto (Phoma spp). Avances Técnicos Cenicafé, 278, 1-4. http://hdl.handle.net/10778/4180

Gómez, A. (1990a). Las malezas nobles previenen la erosión. Avances Técnicos Cenicafé, 151, 1-4. http:/l hdl.handle.net/10778/922

Gómez, A. (1990b). Manejo integrado de malezas en el cultivo del café y la erosión de los suelos. En Centro Nacional de Investigaciones de Café (Ed.), 50 años de Cenicafé 1938-1988: Conferencias conmemorativas. (pp. 15-22). Cenicafé. http://hdl.handle.net/10778/713

Gómez, A., Ramírez, C. J., Cruz, R. G., \& Rivera, J. H. (1987). Manejo y control integrado de malezas en cafetales y potreros de la zona cafetera colombiana. Cenicafé.

Gómez, A., \& Rivera, J. H. (1987). Descripción de arvenses en plantaciones de café. Cenicafé. http://hdl. handle.net/10778/613

González, H. (2013). Identificación de las principales unidades de suelos de la zona cafetera. En Federación Nacional de Cafeteros de Colombia (Ed.), Manual del cafetero colombiano: investigación y tecnología para la sostenibilidad de la caficultura (Vol. 1, pp. 269-283). Cenicafé.

Grime, J. (1973). Competitive exclusion in herbaceous vegetation. Nature, 242, 344-347. https://doi. org/10.1038/242344a0

Haynes, R. J. (2005). Labile Organic Matter Fractions as Central Components of the Quality of Agricultural Soils: An Overview. En Advances in Agronomy (Vol. 85, pp. 221-268). Elsevier. https://doi.org/10.1016/ S0065-2113(04)85005-3

Heap, I. M. (2005). Criteria for Confirmation of Herbicide-Resistant Weeds. International Survey of HerbicideResistant Weeds. http://www.weedscience.org/Pages/ResistanceCriterion.pdf

Herrera O., M. (1983). Expectativas sobre la aplicación de herbicidas en áreas cafeteras de los departamentos de Caldas, Quindio y Risaralda [Tesis de pregrado]. Universidad de Caldas.

Higley, L.G., \& Pedigo, L. P. (1997). The EIL concept. En L.G. Highley, \& L. P. Pedigo(Eds.), Economic thresholds for integrated pest management. (pp. 9-21). University of Nebraska.

Hincapié, E., \& Salazar, L. F. (2007). Manejo integrado de arvenses en la zona cafetera central de Colombia. Avances Técnicos Cenicafé, 359, 1-12. http://hdl.handle.net/10778/379

Holm, L., Plucknett, D., Pancho, J., \& Herberger, J. (1977). The world's worst weeds, distribution and biology. University Press of Hawaii.

186 Manejo agronómico

de los sistemas de producción de café 
Hoyos, J. (1990). Espectro de control y persistencia de la acción de herbicidas (sistémicos y de contacto) y guadañadora, en 20 especies de malezas más frecuentes en cafetales [Tesis de pregrado]. Universidad de Caldas.

Jaramillo, A. (2000). Climatología de región andina de Colombia: Microclima y fenología del cultivo del café. Cenicafé.

Jaramillo, A. (2005a). Clima andino y café en Colombia. Cenicafé.

Jaramillo, A. (2005b). La redistribución de la radiación solar y la lluvia dentro de plantaciones de café (Coffea arabica L.). Revista de la Academia Colombiana de Ciencias Exactas Físicas y Naturales, 29(112), 371-382.

Jaramillo, A. (2016). Manejo integrado de arvenses en la zona cafetera central de Colombia. Avances Técnicos Cenicafé, 465, 1-12. http://hdl.handle.net/10778/703

Jaramillo, A., \& Arcila, J. (1996). Épocas recomendables para la siembra de los cafetos. Avances Técnicos Cenicafé, 229, 1-8. http://hdl.handle.net/10778/4182

Jaramillo, A., \& Chaves, B. (2000). Distribución de la precipitación en Colombia analizada mediante conglomeración estadística. Revista Cenicafé, 51(2), 102-113. http://hdl.handle.net/10778/971

Jaramillo, A., \& Gómez, L. (1989). Microclima en cafetales a libre exposición solar y bajo sombrío. Revista Cenicafé, 40(3), 65-67.http://hdl.handle.net/10778/808

Jaramillo, A., \& Ramírez, V. H., Arcila-Pulgarín, J. (2011a). Patrones de distribución de la lluvia en la zona cafetera. Avances Técnicos Cenicafé, 410, 1-8. http://hdl.handle.net/10778/328

Jaramillo, A., \& Ramírez, V. H., Arcila-Pulgarín, J. (2011b). Distribución de la lluvia: Clave para planificar las labores en el cultivo del café en Colombia. Avances Técnicos Cenicafé, 411, 1-8. http://hdl.handle. net/10778/326

Jiménez, E., \& Golberg, D. (1982) Estudios ecológicos del agroecosistema cafetalero: Efecto de diferentes estructuras vegetales sobre el balance hídrico del cafetal. En E. Jiménez, \& A. Gómez (Eds.), Estudios ecológicos en agroecosistema cafetalero. (pp. 39-54). Continental.

Kropff, M. J., \& Lotz, L. A. P. (1992). Systems approaches to quantify crop-weed interactions and their application in weed management. Agricultural Systems, 40(1), 265-282. https://doi.org/10.1016/0308521X(92)90024-1

Lao, F., Pérez, E., Paredes, E., \& García, R. (1992). Umbrales de daño y económico de Rottboellia cochinchinensis en papa y maíz. Protección de Plantas, 2(4), 53-65. 
Lemes, L. N., Carvalho, L. B., Souza, M. C, \& Alves, P. L. C. A. (2010). Weed interference on coffee fruit production during a four-year investigation after planting. African Journal of Agricultural Research, 5(10), 1138-1143. http://hdl.handle.net/11449/1124

Lin, B. B. (2007). Agroforestry management as an adaptive strategy against potential microclimate extremes in coffee agriculture. Agricultural and Forest Meteorology, 144(1), 85-94. https://doi. org/10.1016/j. agrformet.2006.12.009

Lindquist, L. (2001). Mechanisms of crop loss due to weed competition. En R. K. D. Paterson, \& L. G. Highley (Eds.), Biotic stress and yield loss (pp. 233-253). CRC Press.

Llorens, J. (1999). Poda de formación: Arte, ciencia, paciencia y práctica. https://www.isahispana.com/ portals/O/docs/treecare/poda_de_form.pdf

Lobell, D. B., Cassman, K. G., \& Field, C. B. (2009). Crop Yield Gaps: Their Importance, Magnitudes, and Causes. Annual Review of Environment and Resources, 34(1), 179-204. https://doi.org/10.1146/annurev. environ.041008.093740

Loomis, R. S., \& Connor, D. J. (1992). Crop Ecology: Productivity and Management in Agricultural Systems. Cambridge University Press. https://doi.org/10.1017/CBO9781139170161

Loomis, R. S., Williams, W. A., \& Hall, A. E. (1971). Agricultural Productivity. Annual Review of Plant Physiology, 22(1), 431-468. https://doi.org/10.1146/annurev.pp.22.060171.002243

López, J. A., Villalba-Gault, D. A., Salazar, L. F., \& Cárdenas, O. A. (2012). Manejo integrado de arvenses en el cultivo de café: Nueva alternativa de control químico. Avances Técnicos Cenicafé, 417, 1-8. http://hdl. handle.net/10778/334

Marra, M. C., \& Carlson, G. A. (1983). An Economic Threshold Model for Weeds in Soybeans (Glycine max). Weed Science, 31(5), 604-609. https://doi.org/10.1017/S0043174500070053

Martínez, R. (2009). Sistemas de producción agrícola sostenible. Tecnología en Marcha, 22(2), 23-39. https://revistas.tec.ac.cr/index.php/tec_marcha/article/view/114

Martins, E. P., \& Furlani, E. (2010). Yield performance and leaf nutrient levels of coffee cultivars under different plant densities. Scientia Agricola, 67(6), 720-726. https://doi.org/10.1590/S0103-90162010000600015

Matiello, J. B., García, A. W. R., \& Almeida, S. R. (2007). Recomendando a poda em cafezais. Revista Brasileira de Tecnologia Cafeeira, 4(11), 1-40.

Matthews, G., Brawley, A., \& Hayes, M. (1998). Effect of glyphosate drift on non-glyphosate tolerant corn. Southern Weed Science Society Proceedings, 51, 259-260. 
Mejía, C., Bustillo, A. E., Duque, H., Montoya, E. C. \& Benavides Machado, P. (2007). Análisis biológico y económico del manejo integrado de la broca en la renovación de cafetales. Revista Cenicafé, 58(2), 99110. http://hdl.handle.net/10778/139

Mejía, C. G., Posada, H. E., García, J. C., Chaparro, M. C., Rendón, J. R., Trejos, J. F., Montoya, D. F., Menza, H. D., Sánchez, P. M., Baute, J. E., Torres, J. C., \& Ospina, C. M. (2013). Producción de semilla de variedad Castilloß y sus componentes regionales. En Federación Nacional de Cafeteros de Colombia (Ed.), Manual del cafetero colombiano: investigación y tecnología para la sostenibilidad de la caficultura (Vol. 3, pp. 167-179). Cenicafé.

Menza, H.D. (2006). Evaluación de la resistencia de tres arvenses de la zona cafetera colombiana al glifosato y alternativas para su manejo [Tesis de pregrado]. Universidad Nacional de Colombia

Menza, H.D., \& Peláez, M.J. (2015). Alternativas para el manejo cultural de la muerte descendente del cafeto. Avances Técnicos Cenicafé, 456, 1-8. http://hdl.handle.net/10778/610

Menza, H.D., \& Salazar, L.F. (2006). Estudios de resistencia al glifosato en tres arvenses de la zona cafetera colombiana y alternativas para su manejo. Avances Técnicos Cenicafé, 350, 1-12. http://hdl. handle.net/10778/344

Menza, H. D. \& Salazar, L.F. (2007). Alternativas de control químico para la prevención y manejo de la resistencia de arvenses al glifosato. Revista Cenicafé, 58(2), 91-98. http://hdl. handle.net/10778/221

Mestre, A. (1979). La desyerba en los cafetales produce ganancias. Avances Técnicos Cenicafé, 87, 1-2. http://hdl.handle.net/10778/879

Mestre, A., \& Ospina, H.F. (1994a). Estabilización de la producción en las fincas cafeteras. Avances Técnicos Cenicafé, 200, 1-4. http://hdl.handle.net/10778/1060

Mestre, A., \& Ospina, H.F. (1994b). Manejo de los cafetales para estabilizar la producción en las fincas cafeteras. Avances Técnicos Cenicafé, 201, 1-8. http://hdl.handle.net/10778/1061

Mestre, A., \& Salazar, J. N. (1991). Iniciación de la fertilización en zocas de café. Revista Cenicafé, 42(2), 53- 60. http://hdl.handle.net/10778/4196

Mestre, A., \& Salazar, J. N. (1995). Productividad de siembras nuevas y zocas de café. Avances Técnicos Cenicafé, 215, 1-4. http://hdl.handle.net/10778/1072

Mestre, A., \& Salazar, J. N. (1998). Establecimiento de un sistema de manejo de cafetales. Avances Técnicos Cenicafé, 254, 1-4. http://hdl.handle.net/10778/1047

Montaño, M. I., \& Torres, L. M. (1994). Determinación de la época crítica y umbral de competencia de malezas en el cultivo de alcachofa (Cynara scolymus L.) en la sabana de Bogotá [Tesis de pregrado]. Universidad Distrital Francisco José de Caldas. 
Monteoliva, S., Baroto, J., \& Fernández, M. E. (2015). Anatomía y densidad de la madera en Eucalyptus: variación interespecífica e implicancia en la resistencia al estrés abiótico. Revista de la Facultad de Agronomía, La Plata, 114(2), 209-217.

Moreno, A. M. (2007). Fundamentos sobre sistemas de producción. En J. Arcila, F.F. Farfán, A. M. Moreno, L.F. Salazar, \& E. Hincapié (Eds.), Sistemas de producción de café en Colombia (pp. 15-20). Cenicafé. http://hdl.handle.net/10778/720

Moreno, A. M. (2010). Evite pérdidas económicas al renovar por zoqueo: Resiembre los sitios perdidos. Avances Técnicos Cenicafé, 398, 1-4. http://hdl.handle.net/10778/357

Moreno, A. M., Rendón, J. R., \& Medina, R. D. (2014). Efecto de la población dinámica de tallos sobre la producción de Coffea arabica L. Revista Cenicafé, 65(2), 18-26. http://hdl.handle.net/10778/545

Moreno, A. M., \& Rivera, P. H. (2003). Rotación de cultivos intercalados con café, utilizando el manejo integrado de arvenses. Avances Técnicos Cenicafé, 307, 1-8. http://hdl.handle.net/10778/4184

Moreno, A. M., \& Sánchez, P. M. (2012). Reduzca los costos en el establecimiento del café: Intercale cultivos transitorios. Avances Técnicos Cenicafé, 419, 1-4. http://hdl.handle.net/10778/331

Moreno, G. (2002a) Nueva variedad de café de porte alto resistente a la roya del cafeto. Revista Cenicafé, 53(2), 132-143. http://hdl.handle.net/10778/1021

Moreno, G. (2002b). Tabi: variedad de café de porte alto con resistencia a la roya. Avances Técnicos Cenicafé, 300, 1-4. http://hdl.handle.net/10778/4185

Moreno, A. A. (1980). El herbicida Roundup, nueva alternativa para controlar malezas en cafetales. Nueva Agricultura Tropical, 23, 7-14.

Mortensen, A. D. \& Coble, D. A. (1997). Economic threshold for weeds management. En L.G. Highley, \& L. P. Pedigo(Eds.), Economic thresholds for integrated pest management. (pp. 89-113). University of Nebraska.

Mosquera, L. P., Riaño, N. M., Arcila, J., \& Ponce, C. A. (1999). Fotosíntesis, respiración y fotorrespiración en hojas de café Coffea sp. Revista Cenicafé, 50(3), 215-221. http://hdl.handle.net/10778/4197

Musalem,A.(2001).Asistenciatécnicaycapacitaciónensistemasagroforestalestipomultiestratos(Publicación No. 11/01). Asociación Latinoamericana de Integración (ALADI). http://www.aladi.org/nsfaladi/estudios. nsf/cb5344cb18a6b38903256aa700685717/c8150a32e41c8e3603256a3100634b50/\$FILE/11-01.doc

Njoroge, J. M. (1994a). Advisory notes on management of resistant weeds coffee, Kenya. Kenya Coffee, 59(694), 1821-1823. 
Njoroge, J. M. (1994b). Weeds and weed control in coffee. Experimental Agriculture, 30(4), 421-429. https:/l doi.org/10.1017/S0014479700024662

Njoroge, J. M. \& Mwakha E. (1983). Observations on the effects of weeding and cover crops on coffee yield and quality. Kenya Coffee, 48(569), 219-224.

Oerke, E. C., Dehne, H. W., Schonbeck, F., \& Weber, A. (1994). Crop production and crop protection: Estimated losses in major food and cash crops. Elsevier. https://doi.org/10.1016/C2009-0-00683-7

Pabón, D., Zea, J., León, G., Montealegre, E., Hurtado, G., \& González, C. (1998). La atmósfera, el tiempo y el clima. En P. Leyva (Ed.), El medio ambiente en Colombia. (pp. 38-86). IDEAM.

Paniagua, G., Hernández, C., Rico, F., Domínguez, F. A., Martínez, E., \& Martínez, C. L. (2015). Effect of high intensity led light on the germination and growth of broccoli seedlings (Brassica oleracea L.). Plibotánica, 40, 199-212.

Parker, C., \& Fryer, J. D. (1975). Problemas que presenta el control de malezas, que causan reducciones importantes en los abastecimientos mundiales de alimentos. Boletín Fitosanitario FAO, 23, 83-95.

Parreiras, S., Ferreira, G., Pereira, D., Moreira, F., \& Guimarães, R. J. (2011). Growth, productivity and bienniality of coffee plants according to cultivation spacing. Pesquisa Agropecuária Brasileira, 46(2), 152160. https://doi.org/10.1590/S0100-204X2011000200006

Paulo, E.M.A. y Furlani, E.B. JR. (2010). Yield performance and leaf nutrient levels of coffee cultivars under different plant densities. Scientia Agricola, 67(6), 720-726.

Pavan, M. A., Dias, J. C., Siqueira, R., Androcioli, A., Colozzi, A., \& Balota, E. L. (1999). High coffee population density to improve fertility of an oxisol. Pesquisa Agropecuária Brasileira, 34(3), 459-465. https:/l doi.org/10.1590/S0100-204X1999000300018

Pavlychenko, T. K., \& Harrington, J. B. (1934). Competitive efficiency of weeds and cereal crops. Canadian Journal of Research, 10(1), 77-94.https://doi.org/10.1139/cjr34-006

Perfecto, I., Rice, R. A., Greenberg, R., \& Van der Voort, M. E. (1996). Shade Coffee: A Disappearing Refuge for BiodiversityShade coffee plantations can contain as much biodiversity as forest habitats. BioScience, 46(8), 598-608. https://doi.org/10.2307/1312989

Pimentel, C. (1999). Relações hídricas em dois híbridos de milho sob dois ciclos de deficiência hídrica. Pesquisa Agropecuaria Brasileira, 34(11), 2021-2027. https://doi.org/10.1590/S0100-204X1999001100007

Posada, H. E., Alvarado, G., Cortina, H. A., Solarte, C. R., Duque-Orrego, H., Baldión, J. V., \& Guzmán, O. (2006). La variedad Castillo® El Tambo: para regiones cafeteras de Cauca, Nariño, Huila, Tolima y Valle del Cauca. Avances Técnicos Cenicafé, 344, 1-8. http://hdl.handle.net/10778/390 
Poveda, G. (2004). La hidroclimatología de Colombia: Una síntesis desde la escala inter-decadal hasta la escala diurna. Revista de la Academia Colombiana de Ciencias Exactas, Físicas y Naturales, 28(107), 201- 221.

Primavesi, A. (1984). Manejo ecológico del suelo: la agricultura en regiones tropicales. El Ateneo.

Quiroz, T., \& Hincapié, E. (2007). Pérdidas de suelo por erosión en sistemas de producción de café con cultivos intercalados. Revista Cenicafé, 58(3), 227-235. http://hdl.handle.net/10778/164

Radosevich, S.R. (1987). Methods to study interactions among crops and weeds. Weed Technology, 1(3),190- 198. https://doi.org/10.1017/S0890037X00029523

Ramírez, V. H. (2014). La fenología del café: Una herramienta para apoyar la toma de decisiones. Avances Técnicos Cenicafé, 441, 1-8. http://hdl.handle.net/10778/489

Ramírez, V. H., \& Jaramillo, A. (2009). Relación del índice oceánico de El Niño y la lluvia, en la región andina central de Colombia. Revista Cenicafé, 60(2), 161-172. http://hdl.handle.net/10778/228

Ramírez, V. H. \& Moreno, A. M. (2013). Renovación de cafetales. En Federación Nacional de Cafeteros de Colombia (Ed.), Manual del cafetero colombiano: investigación y tecnología para la sostenibilidad de la caficultura (Vol. 2, pp. 307-318). Cenicafé.

Ramírez, J. E. (1997). Poda y manejo de Coffea arabica L. ICAFE-CICAFE.

Ramos, S., \& Ferreira, O. W. (2001). Determinación de la cantidad y composición química de la biomasa aérea y subterránea de Pinus oocarpa. Revista Escuela Nacional de Ciencias Forestales, 13(2),13-17.

Rendón, J. R. (2016). Sistemas de renovación de cafetales para recuperar y estabilizar la producción. Avances Técnicos Cenicafé, 463, 1-8. http://hdl.handle.net/10778/701

Rendón, J.R., Arcila, J., \& Montoya, E. C. (2008). Estimación de la producción de café con base en los registros de floración. Revista Cenicafé, 59(3), 238-259. http://hdl. handle.net/10778/228

Rendón, J. R., García, J. C., \& Ramírez, C. A. (2015). El redescope: alternativa para recuperar la densidad de tallos en cafetales establecidos con colinos descopados. Avances Técnicos Cenicafé, 451, 1-8. http:ll hdl.handle.net/10778/565

Restrepo, M., \& Rivera, J. H. (1993). Estudio sobre la diversidad de la flora arvense asociada a la zona cafetera colombiana. Cenicafé.

Rice, E.L. (1984). Allelopathy. Academic Press.

Rivera, J. H. (1994). Construya su equipo para aplicación racional de herbicidas y establezca coberturas nobles en su cafetal. Avances Técnicos Cenicafé, 206, 1-8. http://hdl.handle.net/10778/4186 
Rivera, J. H. (1997). Arvenses y su interferencia en el cultivo del café. Avances Técnicos Cenicafé, 237, 1-8. http://hdl.handle.net/10778/4187

Rivera, J. H. (2000). El selector de arvenses modificado. Avances Técnicos Cenicafé, 271, 1-4. http://hdl. handle.net/10778/4188

Rivillas, C. A., \& Castro, A. M. (2011). Ojo de gallo o gotera del cafeto Omphalia flavida. Boletín Técnico Cenicafé, 37, 1-25. http://hdl.handle.net/10778/596

Rivillas, C. A., Serna, C. A., Cristancho, M. A., \& Gaitán, A. (2011). La roya del cafeto en Colombia: Impacto, manejo y costos de control. Boletín Técnico Cenicafé, 36, 1-51. http://hdl.handle.net/10778/594

Rowland, D. D., Reynolds, D.B. J., \& Blackley, H. J. (1999). Corn and cotton response to drift rates of nondesired herbicide applications. Southern Weed Science Society Proceedings, 52, 30.

Sadeghian, S. (2008). Fertilidad del suelo y nutrición del café en Colombia: Guía práctica. Boletín Técnico Cenicafé, 32, 1-44. http://hdl.handle.net/10778/587

Sadeghian, S. (2016). La acidez del suelo una limitante común para la producción de café. Avances Técnicos Cenicafé, 466, 1-8. http://hdl.handle.net/10778/704

Sadeghian, S., \& Duque, H. (2003). Análisis de suelos: importancia e implicaciones económicas en el cultivo del café. Avances Técnicos Cenicafé, 308, 1-8. http://hdl.handle.net/10778/4189

Sadeghian, S., \& Jaramillo, A. (2017). Nutrición de los cafetales en Colombia en escenarios de La Niña. Avances Técnicos Cenicafé, 473, 1-8. http://hdl.handle.net/10778/4190

Sakai, E., Barbosa, E. A., Silveira, J. \& Pires, R. (2013). Coffea arabica (cv. Catuaí) production and bean size under different population arrangements and soil water availability. Engenharia Agrícola, 33(1), 145-156. https://doi.org/10.1590/S0100-69162013000100015

Salamanca, A., \& Sadeghian, S. (2004). La densidad aparente en suelos de la zona cafetera y su efecto sobre el crecimiento del cafeto. Avances Técnicos Cenicafé, 326, 1-8. http://hdl.handle.net/10778/393

Salas, G. (1987). Suelos y ecosistemas forestales con énfasis en América tropical. IICA.

Salazar, J. N. (1975). Efectos de la intensidad de la desyerba sobre la producción de café. [Seminario Cenicafé 1975]. Cenicafé.

Salazar, J. N., \& Mestre, A. (1991). Efecto del zoqueo en la producción de la variedad Colombia. Avances Técnicos Cenicafé, 326, 1-8. http://hdl.handle.net/10778/926

Salazar, L. F. (2013). Prevenga la infestación de la enredadera espinaca en los cafetales. Avances Técnicos Cenicafé, 427, 1-8. http://hdl.handle.net/10778/412 
Salazar, L. F., Arango, J. G., \& Morales, C. S. (2012). Interferencia de coberturas vegetales en la zona de raíces y entre calles del cultivo del café. Revista Cenicafé, 63(2), 50-57. http://hdl.handle.net/10778/535

Salazar, L.F., \& Hincapié, E. (2005). Arvenses de mayor interferencia en los cafetales Avances Técnicos Cenicafé, 194, 1-12. http://hdl.handle.net/10778/406

Salazar, L. F., \& Hincapié, E. (2009). Interferencia de arvenses en diferentes etapas del cultivo del café en la zona cafetera central. Revista Cenicafé, 60(2), 126-134. http://hdl.handle.net/10778/227.

Salazar, L. F., \& Rivera, J. H. (2001). Evaluaciones para incrementar la eficiencia del selector. Cenicafé.

Salazar, M. R., Arcila, J., Riaño, N. M., \& Bustillo, A. E. (1993). Crecimiento y desarrollo del fruto del café y su relación con la broca. Avances Técnicos Cenicafé, 194, 1-4. http://hdl.handle.net/10778/1045

Sanchez, M. (1995). Consideraciones sobre la nutrición mineral y orgánica en los sistemas de producción de café. En Instituto de Pesquisas e Estudos Florestais (Ed.), Seminário internacional de utilização da madeira de eucalipto (pp. 74-91). IPEF/IPT. https://www.ipef.br/publicacoes/anais/anais seminario internacional utilizacao madeira eucalipto serraria.pdf

Scurlock, J., Long, S., Hall, D., \& Coombs, J. (1987). Introducción a las técnicas en fotosíntesis y bioproductividad. En J. Coombs, D. Hall, \& J. Scurlock (Eds.), Técnicas en fotosíntesis y bioproductividad. Editorial Futura.

Secretaría de Estado de Agricultura Pecuaria e Abastecimento. (s.f.). Sistema de informações do agronegócio. Recuperado en enero de 2004 de http://www.agridata.mg.gov.br/

Sivetz, M. (1971). The coffee plant: This evegreen plant needs shade, moisture. Tea and Coffee Trade Journal, 141(5), 16-33.

Soto, L., Perfecto, I., Castillo, J., \& Caballero, J. (2000). Shade effect on coffee production at the northern Tzeltal zone of the state of Chiapas, México. Agriculture, Ecosystems and Environment, 80, 61-69.

Suárez, S. (1998). Características físicas de los suelos del departamento de Risaralda, relacionadas con el uso, manejo y conservación. Avances Técnicos Cenicafé, 257, 1-8. http://hdl.handle.net/10778/4192

Suárez, S. (2000, julio 24-28). Características físicas de los suelos de la zona cafetera colombiana relacionados con el uso y conservación. [Simposio sobre Suelos de la Zona Cafetera Colombiana]. Cenicafé, Manizales, Colombia.

Suárez, S. (2001). La atmósfera del suelo y la productividad del café. Avances Técnicos Cenicafé, 293, 1-4. http://hdl.handle.net/10778/4193

Tabares, E. (1989). Seguimiento y evaluación sobre la aplicación de herbicidas en áreas cafeteras de los departamentos de Caldas, Risaralda y Quindío [Tesis de posgrado]. Universidad Autónoma de Manizales.

194 \begin{tabular}{l|l} 
Manejo aǵronómico \\
de los sistemas de producción de café
\end{tabular} 
Terry, P. J. (1985). Efficacy of glyphosate for weed control in the tropics and sub-tropics. En E. Grossbard, \& D. Atkinson (Eds.), The herbicide glyphosate. (pp. 375-401). Butter worth.

Tinney, F., Aamodt, O., \& Ahlgren, H. (1937). Preliminary report of a study on methods used in botanical analyses of pasture swards. Journal of the American Society of Agronomy, 29(10), 835-840.

Trojer H. (1954). El ambiente climatológico y el cultivo del café en Colombia: problemas, conocimientos actuales y perspectivas. Revista Cenicafé, 5(57), 22-37. http://hdl.handle.net/10778/4198

Trojer, H. (1959). Fundamentos para la zonificación meteorológica y climatológica del trópico especialmente en Colombia. Revista Cenicafé, 10(8), 289-373. http://hdl.handle.net/10778/719

Uribe, A. (1977). Soqueo de cafetales. Avances Técnicos Cenicafé, 66, 1-4. http://hdl. handle.net/10778/864

Uribe, A., \& Mestre, A. (1980). Efecto de la densidad de población y su sistema de manejo sobre la producción de café. Revista Cenicafé, 31(1), 29-51.http://hdl.handle.net/10778/1056

Valencia, G. (1973). Relación entre el índice de área foliar y la productividad del cafeto. Revista Cenicafé, 24(4), 79-89. http://hdl.handle.net/10778/4199

Valverde, B. E., Richies, C. R., \& Caseley, J. C. (2000). Prevención y manejo de malezas resistentes a herbicidas en arroz: experiencias en América central con Echinocloa colona. Cámara de Insumos Agropecuarios de Costa Rica.

Vargas, W.G. (2002). Guía ilustrada de las plantas de las montañas del Quindío y los Andes centrales. Universidad de Caldas.

Vélez, B. E., Jaramillo, A., Chaves, B., \& Franco, M. (2000). Distribución de la floración y la cosecha de café en tres altitudes. Avances Técnicos Cenicafé, 272, 1-4. http://hdl.handle.net/10778/794

Villa Nova, N. A., Favarin, J. L., Angelocci, L. R., \& Dourado-Neto, D. (2002). Estimativa do coeficiente de cultura do cafeeiro em função de variáveis climatológicas e fitotécnicas. Bragantia, 61(1), 81-88. https://doi. org/10.1590/S0006-87052002000100013

Wadsworth, F. H. (1997). Agriculture handbook. Washington State Department of Agriculture

Wrubel, R. P., \& Gressel, J. (1994). Are Herbicide Mixtures Useful for Delaying the Rapid Evolution of Resistance? A Case Study. Weed Technology, 8(3), 635-648. https://www.jstor.org/stable/3988043

Wyse, D. L. (1994). New Technologies and Approaches for Weed Management in Sustainable Agriculture Systems. Weed Technology, 8(2), 403-407. https://www.jstor.org/stable/3988127 
Zimdahl, R. L. (1980). Weed crop competition: A review. Internacional Plant Protection Center. Zimdahl, R.L. (1993). Fundamentals of weed science. Academic Press. 